ARTICLE

\title{
Multivalency transforms SARS-CoV-2 antibodies into ultrapotent neutralizers
}

Edurne Rujas ${ }^{1,2,3}$, Iga Kucharska1, Yong Zi Tan (1) 1, Samir Benlekbir', Hong Cui ${ }^{1}$, Tiantian Zhao ${ }^{4}$, Gregory A. Wasney, ${ }^{1,5}$, Patrick Budylowski ${ }^{6,7}$, Furkan Guvenc 6,8, Jocelyn C. Newton', Taylor Sicard ${ }^{1,2}$, Anthony Semesi ${ }^{1}$, Krithika Muthuraman ${ }^{1}$, Amy Nouanesengsy ${ }^{1,2}$, Clare Burn Aschner (10 1, Katherine Prieto ${ }^{1}$, Stephanie A. Bueler ${ }^{1}$, Sawsan Youssef ${ }^{9}$, Sindy Liao-Chan ${ }^{9}$, Jacob Glanville ${ }^{9}$, Natasha Christie-Holmes ${ }^{6}$, Samira Mubareka10,11, Scott D. Gray-Owen (1) ${ }^{8}$, John L. Rubinstein (1) 1,2,12, Bebhinn Treanor (1) ${ }^{4,13,14} \&$ Jean-Philippe Julien (10) 1,2,4凶

SARS-CoV-2, the virus responsible for COVID-19, has caused a global pandemic. Antibodies can be powerful biotherapeutics to fight viral infections. Here, we use the human apoferritin protomer as a modular subunit to drive oligomerization of antibody fragments and transform antibodies targeting SARS-CoV-2 into exceptionally potent neutralizers. Using this platform, half-maximal inhibitory concentration $\left(\mathrm{IC}_{50}\right)$ values as low as $9 \times 10^{-14} \mathrm{M}$ are achieved as a result of up to 10,000-fold potency enhancements compared to corresponding lgGs. Combination of three different antibody specificities and the fragment crystallizable ( $F c)$ domain on a single multivalent molecule conferred the ability to overcome viral sequence variability together with outstanding potency and IgG-like bioavailability. The MULTi-specific, multiAffinity antiBODY (Multabody or MB) platform thus uniquely leverages binding avidity together with multi-specificity to deliver ultrapotent and broad neutralizers against SARSCoV-2. The modularity of the platform also makes it relevant for rapid evaluation against other infectious diseases of global health importance. Neutralizing antibodies are a promising therapeutic for SARS-CoV-2.

\footnotetext{
${ }^{1}$ Program in Molecular Medicine, The Hospital for Sick Children Research Institute, Toronto, ON, Canada. ${ }^{2}$ Department of Biochemistry, University of Toronto, Toronto, ON, Canada. ${ }^{3}$ Biofisika Institute (CSIC, UPV/EHU) and Department of Biochemistry and Molecular Biology, University of the Basque Country (UPV/EHU), Bilbao, Spain. ${ }^{4}$ Department of Immunology, University of Toronto, Toronto, ON, Canada. ${ }^{5}$ The Structural \& Biophysical Core Facility, The Hospital for Sick Children Research Institute, Toronto, ON, Canada. ${ }^{6}$ Combined Containment Level 3 Unit, University of Toronto, Toronto, ON, Canada. ${ }^{7}$ Institute of Medical Science, University of Toronto, Toronto, ON, Canada. ${ }^{8}$ Department of Molecular Genetics, University of Toronto, Toronto, ON, Canada. ${ }^{9}$ Distributed Bio, South San Francisco, CA, USA. ${ }^{10}$ Department of Laboratory Medicine and Pathobiology, University of Toronto, Toronto, ON, Canada.

${ }^{11}$ Sunnybrook Health Sciences Centre, Toronto, ON, Canada. ${ }^{12}$ Department of Medical Biophysics, University of Toronto, Toronto, ON, Canada.

${ }^{13}$ Department of Cell and Systems Biology, University of Toronto, Toronto, ON, Canada. ${ }^{14}$ Department of Biological Sciences, University of Toronto

Scarborough, Toronto, ON, Canada. ${ }^{凶}$ email: jean-philippe.julien@sickkids.ca
} 
T he continuous threat to public health from respiratory viruses such as the novel SARS-CoV-2 underscores the urgent need to rapidly develop and deploy prophylactic and therapeutic interventions to combat pandemics. Monoclonal antibodies (mAbs) have been used effectively for the treatment of infectious diseases as exemplified by palivizumab for the prevention of respiratory syncytial virus in high-risk infants ${ }^{1}$ or Zmapp, mAb114, and REGN-EB3 for the treatment of Ebola ${ }^{2}$. Consequently, mAbs targeting the Spike (S) protein of SARSCoV-2 have been a focus for the development of biomedical countermeasures against COVID-19. To date, several antibodies targeting the $S$ protein have been identified ${ }^{3-19}$ with bamlanivimab being the first antibody approved in the United States by the Food and Drug Administration (FDA) for the emergency treatment of SARS-CoV-2 in November 2020. Receptor binding domain (RBD)-directed mAbs that interfere with binding to angiotensin converting enzyme 2 (ACE2), the receptor for cell entry $^{20}$, are usually associated with the highest neutralization potencies $6,18,19$.

mAbs can be isolated by B-cell sorting from infected donors, immunized animals, or by identifying binders in preassembled libraries. Despite these methodologies being robust and reliable for the discovery of virus-specific mAbs, identification of the best antibody clone is usually associated with a time-cost penalty. In addition, RNA viruses have higher mutations rates than DNA viruses and such mutations can significantly alter the potency of neutralizing antibodies. Indeed, several studies have already shown a reduction in neutralization potency from convalescent serum and resistance of certain $\mathrm{mAbs} \mathrm{s}^{21-23}$ to the more recent B.1.1.7 ${ }^{24}, \mathrm{~B} .1 .351^{25}$, and B.1.1.28 $8^{26,27}$ variants of SARS-CoV-2. Hence, there is an unmet need for the development of a platform that bridges antibody discovery and the rapid identification and deployment of highly potent neutralizers less susceptible to viral sequence variability.

The potency of an antibody is greatly affected by its ability to simultaneously interact multiple times with its epitope ${ }^{28-30}$. This enhanced apparent affinity, known as avidity, has been previously reported to increase the neutralization potency of nanobodies 31,32 and of IgGs over Fabs ${ }^{8,10,16}$ against SARS-CoV-2. To leverage the full power of binding avidity, we have developed an antibodyscaffold technology using the human apoferritin protomer as a modular subunit to multimerize antibody fragments and propel mAbs into ultrapotent neutralizers against SARS-CoV-2. Indeed, the resulting Multabody molecules can increase potency by up to four orders of magnitude over corresponding IgGs. In addition, we demonstrate the ability of this technology to combine three different Fab specificities to better overcome point mutations in the Spike. The Multabody offers a versatile IgG-like "plug-andplay" platform to enhance antiviral characteristics of mAbs against SARS-CoV-2, and demonstrates the power of avidity as a mechanism to be leveraged against viral pathogens.

\section{Results}

Avidity enhances neutralization potency. We used the selfassembly of the light chain of human apoferritin to multimerize antigen binding moieties targeting the SARS-CoV-2 S glycoprotein. Apoferritin protomers self-assemble into an octahedrally symmetric structure with an $\sim 6 \mathrm{~nm}$ hydrodynamic radius ( $\mathrm{Rh}$ ) composed of 24 identical polypeptides ${ }^{33}$. The $\mathrm{N}$ terminus of each apoferritin subunit points outwards of the spherical nanocage and is therefore accessible for the genetic fusion of proteins of interest. Upon folding, apoferritin protomers act as building blocks that drive the multimerization of the 24 proteins fused to their $\mathrm{N}$ termini (Fig. 1a).

First, we investigated the impact of multivalency on the ability of the single chain variable domain $\mathrm{VHH}-72$ to block viral infection. VHH-72 has been previously described to neutralize SARS-CoV-2 when fused to a Fc domain, but not in its monovalent format ${ }^{31}$. The light chain of human apoferritin displaying 24 copies of VHH-72 assembled into monodisperse, well-formed spherical particles (Fig. 1b, c) and showed an enhanced binding avidity to the $S$ glycoprotein (Fig. 1d) in comparison to bivalent VHH-72-Fc. Strikingly, display of VHH72 on the light chain of human apoferritin achieved a $\sim 10,000$ fold increase in neutralization potency against SARS-CoV-2 pseudovirus (PsV) compared to the conventional $\mathrm{Fc}$ fusion (Fig. 1e), demonstrating the power of avidity to transform binding moieties into potent neutralizers.

Multabodies have IgG-like properties. The Fc confers IgGs in vivo half-life and effector functions through interaction with neonatal $F_{c}$ receptor $\left(F_{c} R n\right)$ and $F_{c}$ gamma receptors $\left(F_{c} \gamma R\right)$, respectively. To confer these IgG-like properties to our multimeric scaffold, we next sought to incorporate both binding moieties and Fc domains. Because a Fab is a hetero-dimer consisting of a light and a heavy chain, and the Fc is a homodimer, we created singlechain Fab (scFab) and single-chain $\mathrm{Fc}(\mathrm{scFc})$ polypeptide constructs. scFab and $\mathrm{scFc}$ domains were directly fused to the $\mathrm{N}$ terminus of the apoferritin protomer. For in vivo proof-ofprinciple experiments, we generated a species-matched surrogate molecule that consists of mouse light chain apoferritin fusions to a mouse scFab and a mouse scFc (IgG2a subtype). Binding kinetics showed that the resulting MB molecule binds mouse FcRn in a $\mathrm{pH}$ dependent manner-binding at endosomal $\mathrm{pH}$ (5.6) and no binding at physiological $\mathrm{pH}(7.4)$-similar to the parental IgG (Fig. 2a). Expectedly, binding to the high-affinity mouse Fc $\gamma \mathrm{R} 1$ was enhanced through avidity effects in comparison to the parental IgG. Hence, we generated a modified mouse scFc version that includes the $\mathrm{Fc} \gamma \mathrm{R}$-silencing mutations LALAP to lower Fc binding in a multimeric context (Fig. 2a). Subcutaneous administration of $\mathrm{MBs}$ in $\mathrm{C} 57 \mathrm{BL} / 6$ or $\mathrm{BALB} / \mathrm{c}$ mice was well tolerated with no decrease in body weight or visible adverse events. The MB showed favorable IgG-like serum half-life (Fig. 2b), with a prolonged detectable titer in the sera for the lower Fc $\gamma$ R-binding $\mathrm{MB}$ (LALAP Fc sequence) compared to the WT MB, indicative of a role for the $\mathrm{Fc}$ in dictating in vivo bioavailability. Live $2 \mathrm{D}$ and $3 \mathrm{D}$ imaging revealed that the fluorescently-labeled MB biodistributed systemically like the corresponding IgG, without accumulation in any specific tissue (Fig. 2c and Fig. S1). In contrast, $15 \mathrm{~nm}$ gold nanoparticles (GNP), which have a similar Rh as MBs, rapidly disseminated from the site of injection (Fig. 2c and Fig. S1). Presumably because all sequences derived from the host, the surrogate mouse $\mathrm{MB}$ did not induce an anti-drug antibody response in mice (Fig. 2d), thus further highlighting the IgG-like properties of the MB platform.

Protein engineering to achieve higher valency. In view of these favorable results for a mouse MB surrogate, we aimed to generate fully-human $\mathrm{MBs}$ derived from the previously reported IgG BD23 ${ }^{12}$ and IgG $4 \mathrm{~A}^{13}$ that target the SARS-CoV-2 spike RBD and N-terminal domain (NTD), respectively. Addition of scFcs into the $\mathrm{MB}$ reduces the number of scFabs that can be multimerized. In order to endow the MB platform with Fc without compromising Fab avidity and hence neutralization potency, we engineered the apoferritin protomer to accommodate more than 24 components per particle. Based on its four-helical bundle fold, the human apoferritin protomer was split into two halves: the two $\mathrm{N}$-terminal a helices (N-Ferritin) and the two C-terminal a helices (C-Ferritin). In this configuration, the scFc fragment of human IgG1 and the scFab of anti-SARS-CoV-2 IgGs were genetically fused at the $\mathrm{N}$ terminus of each apoferritin half, 
a

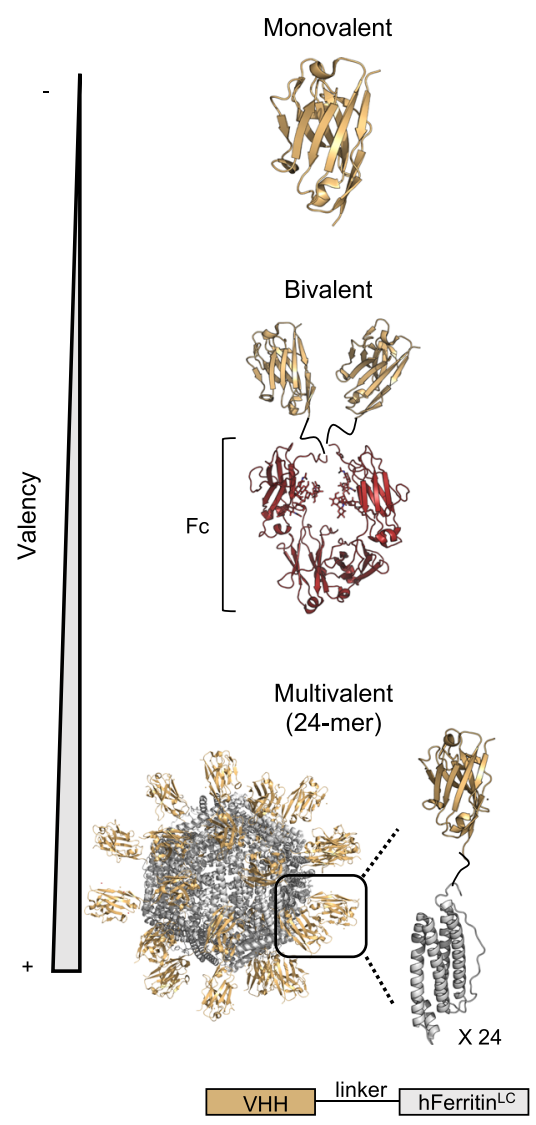

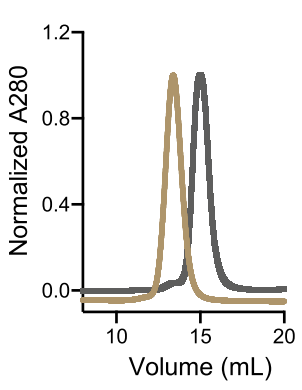

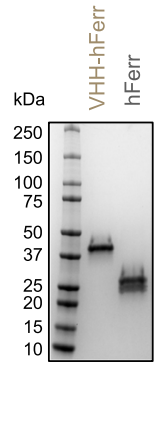

C
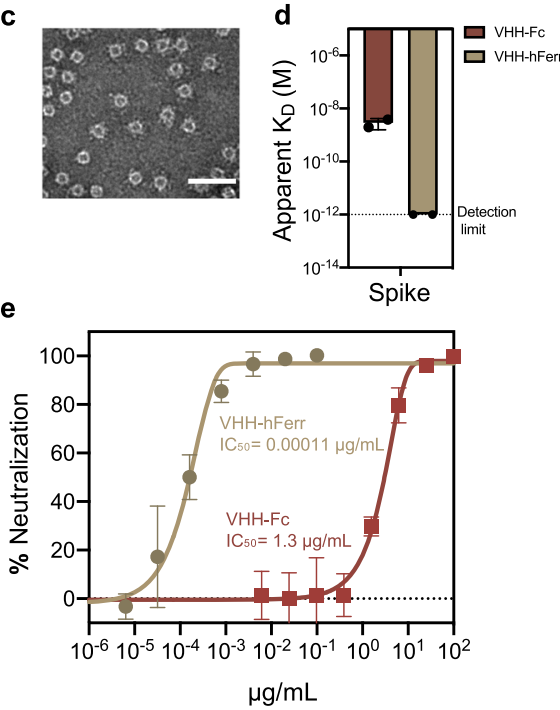

Fig. 1 Avidity enhances binding and neutralization of VHH against SARS-CoV-2. a Schematic representation of a monomeric VHH domain and its multimerization using a conventional Fc (dark red) scaffold or human apoferritin (gray). b Size exclusion chromatography and SDS-PAGE of apoferritin alone (gray) and $\mathrm{VHH}-72$ apoferritin particles (gold). c Negative stain electron microscopy of $\mathrm{VHH}-72$ apoferritin particles. (Scale bar $50 \mathrm{~nm}$, representative of two independent experiments). d Comparison of the binding avidity (apparent $K_{\mathrm{D}}$ ) of VHH-72 to SARS-CoV-2 S protein when displayed in a bivalent (dark red) or 24-mer (gold) format. Bars indicate the mean values of $n=2$ biologically independent experiments. Apparent $K_{\mathrm{D}}$ lower than 10 $-12 \mathrm{M}$ (dash line) is beyond the instrument detection limit. e Neutralization potency against SARS-CoV-2 PsV (color coding is as in (d)). One representative out of two biologically independent replicates with similar results is shown. Mean values \pm SD of two technical replicates is represented in the plot. Median $\mathrm{IC}_{50}$ values of the two biologically independent replicates are shown. Source data are provided as a Source Data file.

respectively. Split apoferritin complementation led to heterodimerization of the two halves and consequently resulted in a very efficient hetero-dimerization process of the fused proteins. Co-expression of the scFab-C-Ferritin and scFc-N-Ferritin genes together with the scFab-Ferritin gene in excess resulted in a full apoferritin self-assembly that displays high numbers of scFab and low numbers of scFc on the nanocage periphery (Fig. 3a and Materials and Methods). Conveniently, this design allows for the straightforward purification of the $\mathrm{MB}$ using Protein $\mathrm{A}$ akin to IgG purification.

This split MB design forms $16 \mathrm{~nm} \mathrm{Rh}$ spherical particles with an uninterrupted ring of density and regularly spaced protruding scFabs and scFc (Fig. 3b, c). Hence, the MB is on the lower size range of natural IgMs ${ }^{34}$, but packs more weight on a similar size to achieve high multi-valency. Binding kinetics experiments demonstrated that high binding avidity of the MB for the Spike was preserved upon addition of Fc fragments (Fig. 3d). Binding to human $\mathrm{Fc} \gamma \mathrm{RI}$ and $\mathrm{FcRn}$ at endosomal $\mathrm{pH}$ confirmed that $\mathrm{scFc}$ was properly folded in the split $\mathrm{MB}$ design. In addition, the LALAP mutations in the scFc lowered the binding affinity to human Fc $\gamma$ RI (Fig. 3e), as previously observed with the surrogate mouse MB (Fig. 2a). SARS-CoV-2 PsV neutralization assays with the split design MBs showed that enhanced binding affinity for the Spike translates into an improved neutralization potency in comparison to their IgG counterparts, with a 1600 -fold and $>2000$-fold increase for BD23 and 4A8, respectively (Fig. 3f). Combined, this data supported the further exploration of the MB as an IgG-like platform that confers exquisite binding avidity and PsV neutralization across epitopes on different Spike domains.

From antibody discovery to ultrapotent neutralizers. We next assessed the ability of the MB platform to transform mAb binders identified from initial phage display screens into potent neutralizers against SARS-CoV-2 (Fig. 4a). Following standard biopanning protocols against the RBD of SARS-CoV-2, 20 human $\mathrm{mAb}$ binders with moderate affinities that range from $10^{-6}$ to $10^{-}$ ${ }^{8} \mathrm{M}$ were selected (Supplementary Data 1 ). These mAbs were produced as full-length IgGs and MBs and their capacity to block viral infection was compared in a neutralization assay against SARS-CoV-2 PsV (Fig. 4b and Fig. S2a). Notably, MB expression yields, homogeneity and thermostability was similar to those of the parental IgG (Fig. S3) and the MB enhanced the potency of 18 out of $20(90 \%)$ IgGs by up to four orders of magnitude. The largest increment was observed for $\mathrm{mAb} 298$ which went from a mean $\mathrm{IC}_{50}$ of $\sim 0.3 \mu \mathrm{g} / \mathrm{mL}$ as an $\mathrm{IgG}$ to $0.0001 \mu \mathrm{g} / \mathrm{mL}$ as a $\mathrm{MB}$. Strikingly, $11 \mathrm{mAbs}$ were converted from non-neutralizing IgGs to neutralizing MBs in the tested concentration ranges. Seven 
a

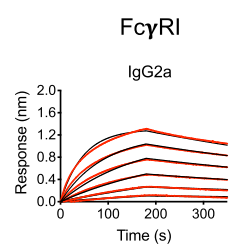

WT MB

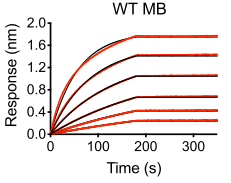

LALAP MB

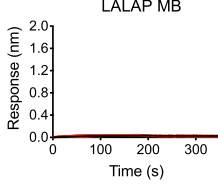

Bioavailability

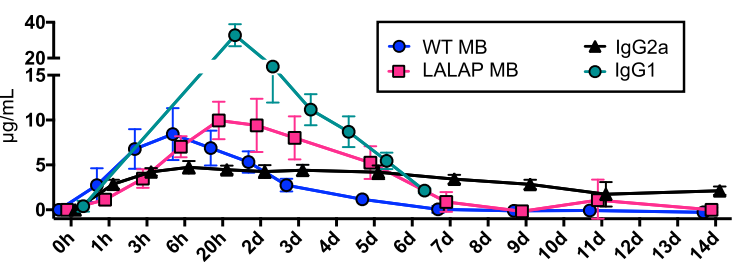

WT MB

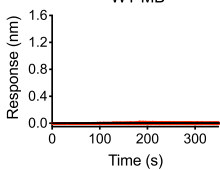

c PBS
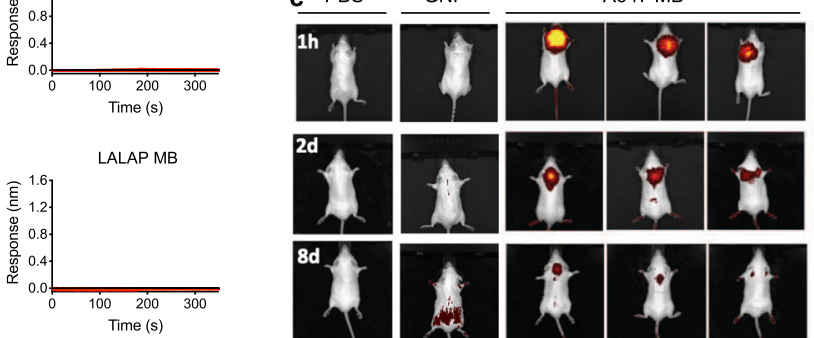
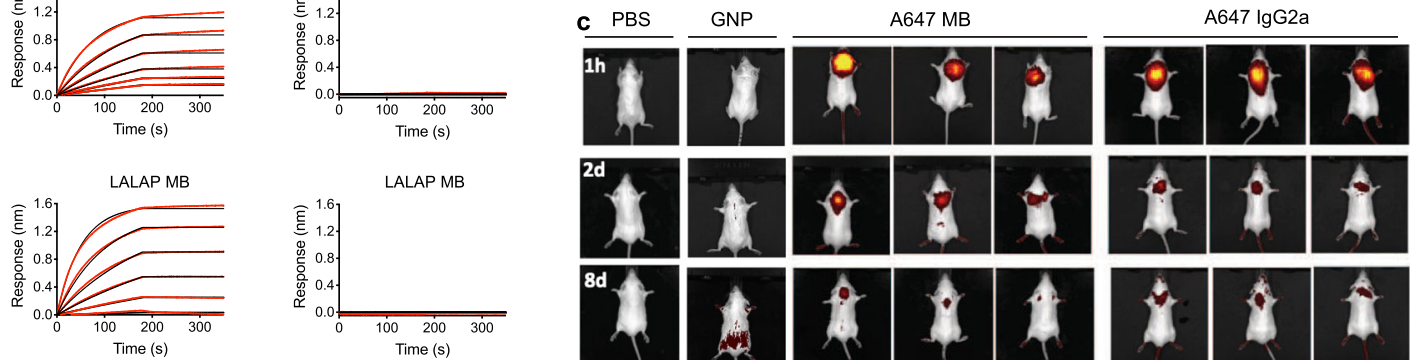

Anti-drug-antibodies

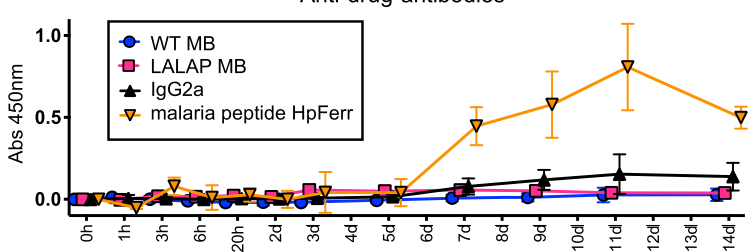

Fig. 2 Bioavailability, biodistribution, and immunogenicity of a mouse surrogate Multabody. a Binding kinetics of WT and Fc-modified (LALAP mutation) $\mathrm{MB}$ to mouse FcyRI (left) and mouse FcRn at endosomal (middle) and physiological (right) pH in comparison to the parental lgG. Two-fold dilution series from 100 to $3 \mathrm{nM}$ (IgG) and 10 to $0.3 \mathrm{nM}(\mathrm{MB})$ were used. Red lines represent raw data; black lines represent global fits. b Five male C57BL/6 mice per group were used to assess the serum concentration of a surrogate mouse MB, a Fc-modified MB (LALAP mutation), and parental mouse IgGs (IgG1 and IgG2a subtypes) after subcutaneous administration of $5 \mathrm{mg} / \mathrm{kg}$. c MB and lgG2a samples were labeled with Alexa-647 for visualization of their biodistribution post subcutaneous injection into three male BALB/c mice/group via live noninvasive $2 \mathrm{D}$ whole body imaging. $15 \mathrm{~nm}$ fluorescently-labeled gold nanoparticles (GNP), which have a similar Rh value as the Multabody are shown as a comparator. d Five male C57BL/6 mice per group were used to assess any anti-drug-antibody response induced by the mouse surrogate Multabody in comparison to parental IgG and a species-mismatched malaria PfCSP peptide fused to Helicobacter pylori ferritin (HpFerr). Mean values \pm SD of $n=5$ mice is shown in (b) and (d). Source data of panels $\mathbf{b}$ and $\mathbf{d}$ are provided as a Source Data file.

MBs displayed exceptional potency with $\mathrm{IC}_{50}$ values between $0.2-2 \mathrm{ng} / \mathrm{mL}$ against SARS-CoV-2 PsV using two different target cells (293T-ACE2 and HeLa-ACE2 cells; Fig. 4b and Fig. S2b). PsV neutralization assays using recombinant mAbs REGN10933 and REGN10987 as benchmark showed similar IC $_{50}$ values $(0.0044$ and $0.030 \mu \mathrm{g} / \mathrm{mL}$, respectively) to those previously reported $^{8}$, and thus confirmed the extraordinary potency of the MBs observed in our assays. The enhanced neutralization potency of the MB was further confirmed with authentic SARS-CoV-2 virus for the mAbs with the highest potency (Fig. 4c and Fig. S2c), as also benchmarked with the two recombinant REGN mAbs. The less sensitive neutralization phenotype we observed against authentic virus in comparison to $\mathrm{PsV}$ is also in agreement with previous reports ${ }^{5,6,9,12}$

Retrospectively, all IgGs and MBs were tested for their ability to bind to the Spike glycoprotein and the RBD of SARS-CoV-2 (Fig. S4). Increased avidity resulted in higher apparent binding affinities with no detectable off-rates against the Spike glycoprotein, most likely due to inter-spike crosslinking that translates into high neutralization potency (Fig. 4b-d and Fig. S4). Overall, the data show that the MB platform is compatible with rapid delivery of ultrapotent IgG-like molecules even when starting with mAbs of modest neutralization characteristics.
Epitope mapping. Based on their neutralization potency, seven mAbs were selected for further characterization: 298 (IGHV1-46/ IGKV4-1), 82 (IGHV1-46/IGKV1-39), 46 (IGHV3-23/IGKV139), 324 (IGHV1-69/IGKV1-39), 236 (IGHV1-69/IGKV2-28), 52 (IGHV1-69/IGKV1-39), and 80 (IGHV1-69/IGKV4-1) (Fig. 4b and Supplementary Data 1). Epitope binning experiments showed that these mAbs target two main sites on the RBD, with one of these bins overlapping with the ACE2 binding site (Fig. 5a and Fig. S5). Cryo-EM structures of Fab-SARS-CoV-2 S complexes at a global resolution of $\sim 6-7 \AA$ confirmed that mAbs 324,298 , and 80 bind overlapping epitopes (Fig. 5b, Fig. S6a-c, and Table S1). To gain insight into the binding of mAbs targeting the other bin, we obtained the cryo-EM structure of Fab 46 in complex with the RBD at a global resolution of $4.0 \AA$ (Fig. 5c, Fig. S6d, and Table S1), and the crystal structure of Fabs 298 and 52 as a ternary complex with the RBD at $2.95 \AA$ resolution (Fig. 5d, Fig. S7, and Table S2).

The crystal structure shows that Fab 298 binds almost exclusively to the ACE2 receptor binding motif (RBM) of the RBD (residues 438-506). In fact, out of 16 RBD residues involved in binding Fab 298, 12 are also involved in ACE2-RBD binding (Fig. S7a-c and Table S3). The RBM is stabilized by 11 hydrogen bonds from heavy and light chain residues of Fab 298. In addition, RBM Phe486 is contacted by 11 Fab 298 residues 


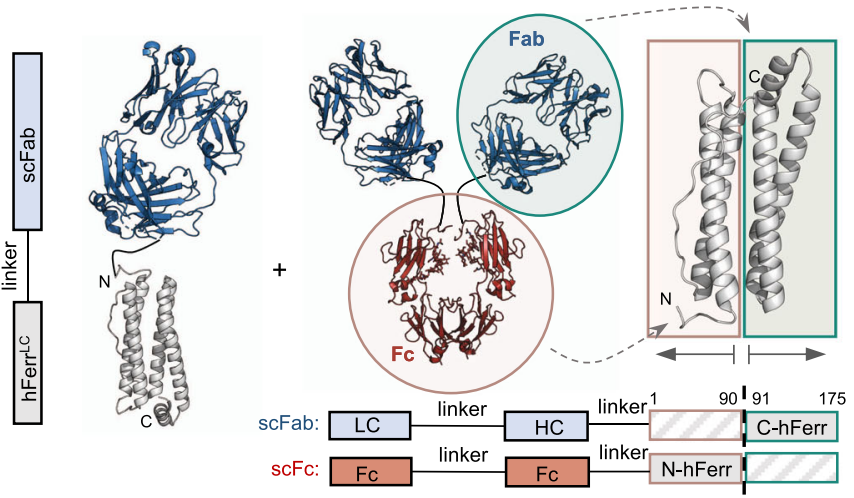

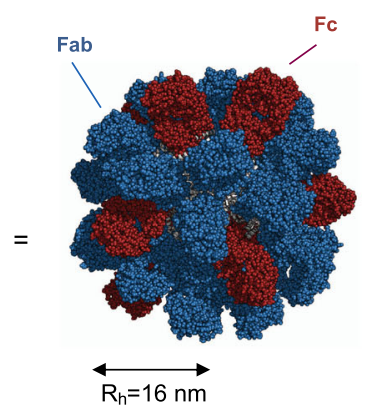
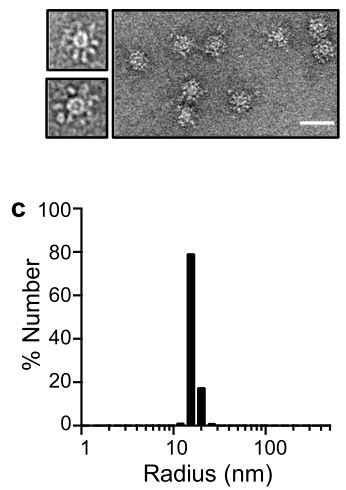

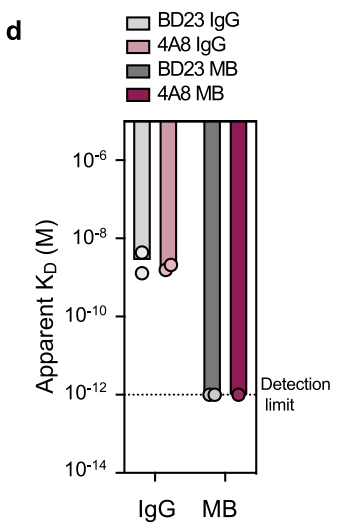

e
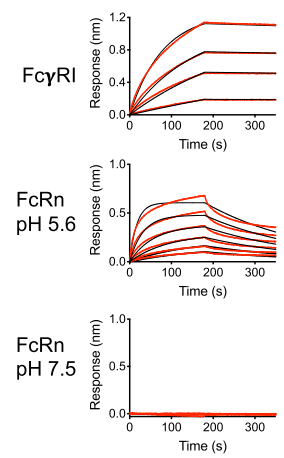

WT MB
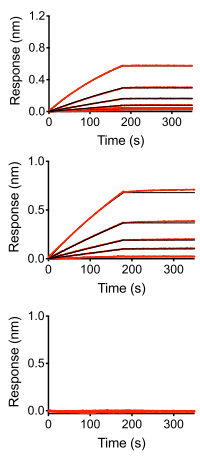

LALAP MB
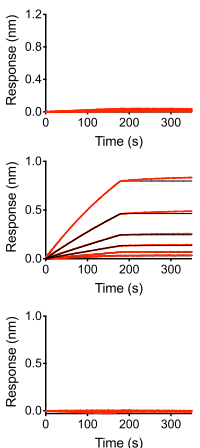

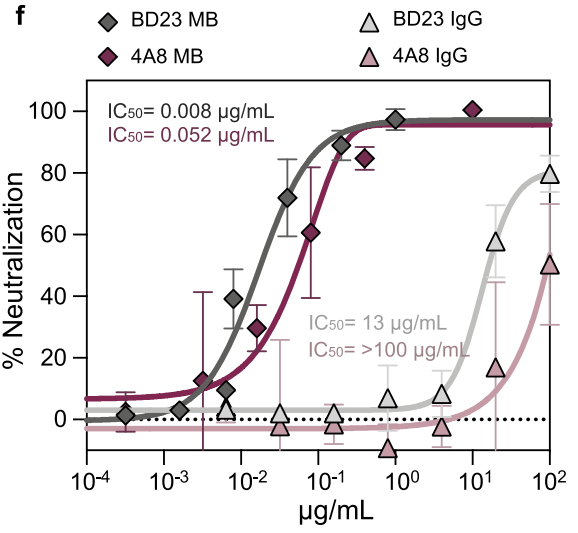

Fig. 3 Protein engineering to multimerize IgG-like particles against SARS-CoV-2. a Schematic representation of the human apoferritin split design. b Negative stain electron micrograph of the MB. (Scale bar $50 \mathrm{~nm}$, representative of two independent experiments). c Hydrodynamic radius (Rh) of the MB. d Avidity effect on the binding (apparent $K_{D}$ ) of $4 A 8$ (purple) and BD23 (gray) to the SARS-CoV-2 Spike. e Sensograms of BD23 lgG and MB with different Fc sequence variants binding to $\mathrm{Fc} \gamma \mathrm{RI}$ (top row), FcRn at endosomal pH (middle row) and FcRn at physiological pH (bottom row). Red lines represent raw data whereas black lines represent global fits. $\mathbf{f}$ Neutralization of SARS-CoV-2 PsV by 4A8 and BD23 IgGs and MBs. Representative data of three biologically independent samples. The mean values \pm SD for two technical replicates is shown in each neutralization plot. Median IC ${ }_{50}$ values of the three biologically independent replicates are indicated. Source data are provided as a Source Data file.

burying $\sim 170 \AA^{2}$ (24\% of the total buried surface area on RBD) and hence is central to the antibody-antigen interaction (Fig. S7a and Table S3).

Detailed analysis of the RBD-52 Fab interface reveals that the epitope of $\mathrm{mAb} 52$ is shifted towards the core of the RBD encompassing 20 residues of the RBM and seven residues in the core domain (Fig. 5c, Fig. S7b, and Table S3). In agreement with the competition data, antibody 52 and antibody 46 share a similar binding site, although they approach the RBD with slightly different angles (Fig. 5c, d and Fig. S7d). Inspection of previously reported structures of $\mathrm{RBD}$-antibody complexes reveal that antibodies 46 and 52 target a site of vulnerability on the SARS$\mathrm{CoV}-2$ spike that has not been described previously (Fig. 5e). The epitope targeted by these antibodies is partially occluded by the NTD in the S "closed" conformation, suggesting that the mechanism of action for this class of antibodies could involve Spike destabilization. Together, these data demonstrate that the enhanced neutralization potency observed for the MB platform through avidity is associated with mAbs that can target distinct epitope bins on the RBD.

Multabodies overcome Spike sequence variability. To explore whether MBs could potentially resist viral escape via their enhanced binding avidity, we tested the effect of four naturally occurring RBD mutations ${ }^{35}$ on the binding and neutralization of the seven human mAbs of highest potency: L452R-located within the epitope of antibodies 46 and 52 (bin 1), A475V and V483A-located within the ACE2 binding site (bin 2), and the circulating $\mathrm{RBD}$ variant $\mathrm{N}_{4} 39 \mathrm{~K}^{36}$ (Fig. $6 \mathrm{a}-\mathrm{c}$ ). In addition, the impact of mutating Asn234-an N-linked glycosylation site-to Gln was also assessed because the absence of glycosylation at this site has been previously reported to decrease sensitivity to neutralizing antibodies targeting the $\mathrm{RBD}^{35}$. The more infectious PsV variant $\mathrm{D} 614 \mathrm{G}^{37}$ was also included in the panel. As expected, mutation L452R significantly decreased binding and potency of mAbs 52 and 46, while antibody 298 was sensitive to mutation A475V (Fig. 6b, c). Deletion of the N-linked glycan at position Asn234 increased viral resistance to the majority of the antibodies, especially mAbs 46, 80, and 324, emphasizing the importance of glycans in viral antigenicity (Fig. 6c). Strikingly, the following antibody specificities in the MB format were minimally impacted in their exceptional neutralization potency by any $\mathrm{S}$ mutation: 298, 80, 324, and 236 (Fig. 6d). Mutation L452R decreased the sensitivity of the $46-\mathrm{MB}$ and $52-\mathrm{MB}$ but in contrast to their parental IgGs, they remained neutralizing against this PsV variant (Fig. 6d). The more infectious SARS-CoV-2 PsV variant D614G was neutralized with similar potency as the WT PsV for both IgGs and MBs (Fig. 6c and Fig. S8a).

$\mathrm{MB}$ cocktails consisting of three monospecific MBs resulted in pan-neutralization across all PsV variants without a significant loss in potency and hence achieved a 100-1000-fold higher potency compared to the corresponding IgG cocktails (Fig. 6e and 
a

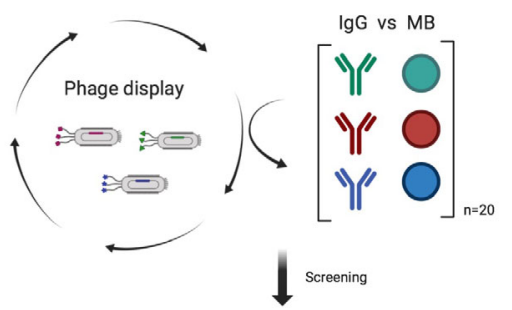

PsV Neutralization/ 293T-ACE2 cells

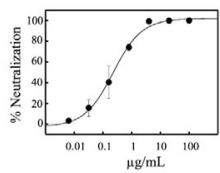

C

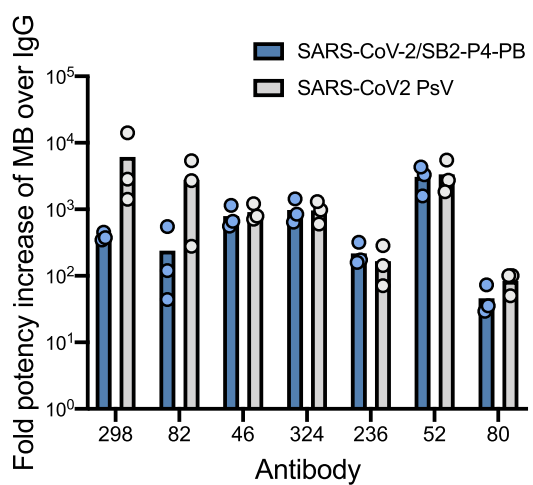

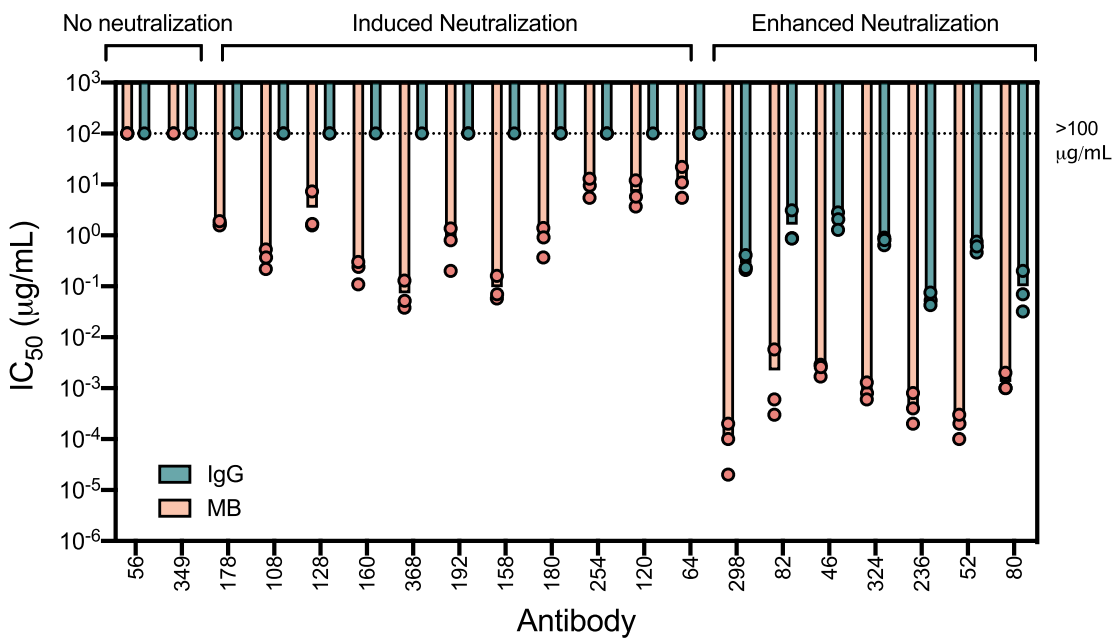

d
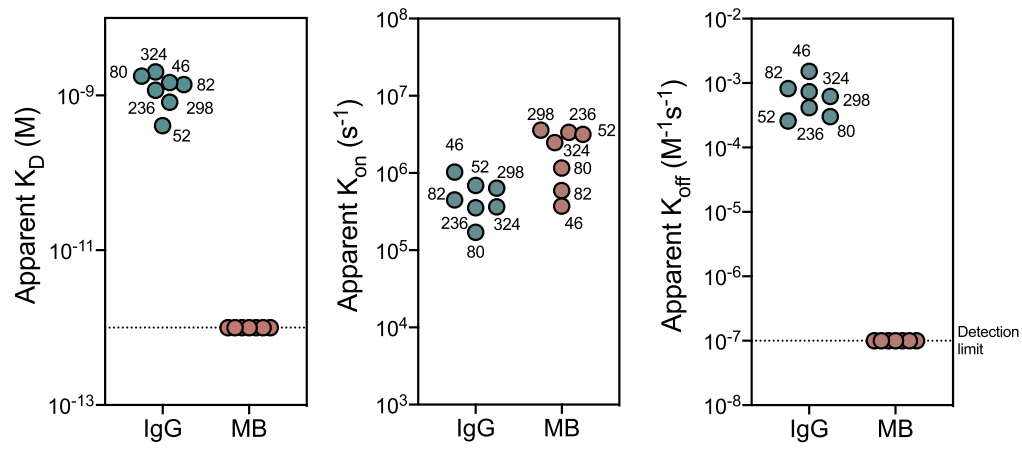

Fig. 4 The Multabody enhances the potency of human mAbs from phage display. a Work flow for the identification of potent anti-SARS-CoV-2 neutralizers using the MB technology. Created with Biorender. b Comparison of neutralization potency between lgGs (cyan) and MBs (pink) that display the same human Fab sequences derived from phage display. $\mathbf{c} I C_{50}$ values fold increase upon multimerization. $\mathbf{d}$ Apparent affinity $\left(K_{D}\right)$, association $\left(k_{o n}\right)$, and dissociation $\left(k_{\text {off }}\right)$ rates of the most potent neutralizing MBs (pink) compared to their lgG counterparts (cyan) for binding the SARS-CoV-2 S protein. Three biological replicates and their mean are shown for $I C_{50}$ values in (b) and (c). Source data of panels $\mathbf{b}-\mathbf{d}$ are provided as a Source Data file.

Fig. S8c, d). In order to achieve breadth within a single molecule, we next generated tri-specific MBs by combining multimerization subunits displaying three different Fabs in the same MB assembly (Fig. S8b). Notably, the resulting tri-specific MBs exhibited panneutralization while preserving the exceptional neutralization potency of the monospecific versions including against the B.1.351 PsV variant (Fig. 6e, f and Fig. S8c, d). The highest potency was observed for the 298-324-46 combination (Fig. S8c, e), where the tri-specific $\mathrm{MB}$ achieved exceptional potency beyond that observed for some of the most potent IgGs reported to date and that we generated recombinantly from available sequences (Fig. 6g). In addition, the $\mathrm{MB}$ format was able to increase the potency of these previously reported highly potent IgGs by a further one to two orders of magnitude against PsV and live replicating SARS-CoV-2 virus (Fig. 6h), thus highlighting the plug-and-play nature of the $\mathrm{MB}$ and the ability of multivalency to enhance the neutralization capacity of mAbs across a range of potencies.

\section{Discussion}

In this study, we reveal how binding avidity can be leveraged as an effective mechanism to propel antibody neutralization potency and resistance from viral mutations. To this effect, we used protein engineering to develop a plug-and-play antibodymultimerization platform that increases avidity of mAbs targeting SARS-CoV-2. The seven most potent $\mathrm{MBs}$ have $\mathrm{IC}_{50}$ values of 0.2 to $2 \mathrm{ng} / \mathrm{mL}\left(9 \times 10^{-14}\right.$ to $\left.9 \times 10^{-13} \mathrm{M}\right)$ against SARS-CoV-2 PsVs and therefore are, to our knowledge, within the most potent antibody-like molecules reported to date against SARS-CoV-2.

The MB platform was designed to include key favorable attributes from a developability perspective. First, the ability to augment antibody potency is independent of antibody sequence, format or epitope targeted. The modularity and flexibility of the platform was exemplified by enhancing the potency of a $\mathrm{VHH}$ and multiple Fabs that target non-overlapping regions on two SARS-CoV-2 S sub-domains (RBD and NTD). Using the MB to enhance the potency of $\mathrm{VHH}$ domains could provide particular value to this class of molecules since its small size allows highly efficient multimerization. Second, in contrast to other approaches that enhance avidity through tandem fusions of single chain variable fragments ${ }^{38,39}$, MBs do not suffer from low stability and in fact self-assemble into highly stable particles with aggregation temperatures similar to those of their parental IgGs. Third, alternative multimerization strategies like streptavidin ${ }^{40}$, verotoxin B subunit scaffolds ${ }^{41}$, or viral-like nanoparticles ${ }^{42}$ face immunogenicity challenges and/or poor bioavailability because of the absence of a Fc fragment and therefore the inability to undergo FcRn-mediated recycling. The light chain of apoferritin is fully human, biologically inactive, has been engineered to 
a

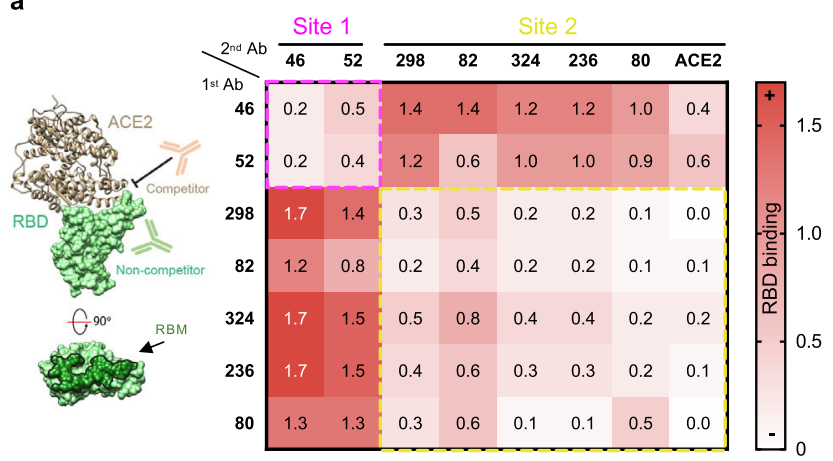

b

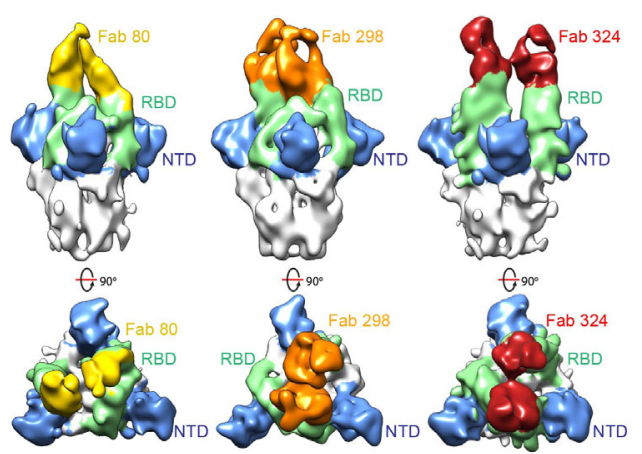

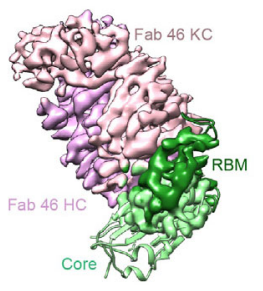

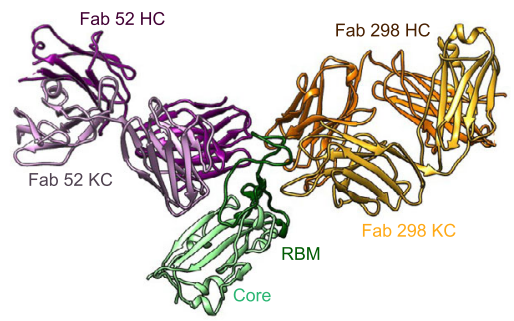

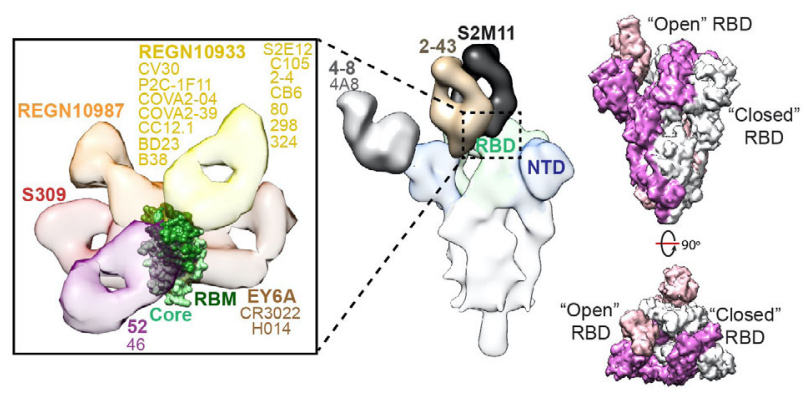

Fig. 5 Epitope delineation of the most potent mAb specificities. a Surface and cartoon representation of RBD (light green for the core and dark green for RBM) and ACE2 ${ }^{66}$ (light brown) binding. Heat map showing binding competition experiments. High signal responses (red) represent low competition while low signal responses (white) correspond to high competition. Epitope bins are highlighted by dashed-line boxes. b $15.0 \AA$ filtered cryo-EM reconstruction of the Spike (gray) in complex with Fab 80 (yellow), 298 (orange), and 324 (red). The RBD and NTD are shown in green and blue, respectively. c Cryo-EM reconstruction of the Fab 46 (pink) and RBD (green) complex. A RBD 66 secondary structure cartoon is fitted into the partial density observed for the RBD. d Crystal structure of the ternary complex formed by Fab 52 (purple), Fab 298 (orange), and RBD (green). e Composite image depicting the side and top view of the unliganded (PDB 6XM4) and the antibody-bound SARS-CoV-2 spike with available PDB or EMD entries $3,4,9,10,13,15,17,67-72$. Inset: close up view of antibodies targeting different antigenic sites on the RBD. The mAb with the lowest reported IC $C_{50}$ value against SARS-CoV-2 PsV was selected as a representative antibody of the bin (highlighted in bold) and those antibodies with similar binding epitopes are listed in the same color below (color coding of Spike, NTD and RBD as in (b)). Individual protomers in the unliganded spike are shown in white, pink, and purple.

include Fc domains, and despite multimerization of $>24 \mathrm{Fab} / \mathrm{Fc}$ fragments, has a Rh similar to an IgM. As such, a surrogate mouse MB did not elicit antidrug antibodies in mice and similar to its parental IgG was detectable in the sera for over a week. However, in vivo bioavailability of the MB was dependent on its binding affinity to $\mathrm{Fc} \gamma \mathrm{Rs}$, suggesting that $\mathrm{Fc}$ avidity will need to be carefully fine-tuned for efficient translation of the $\mathrm{MB}$ to the clinic. In addition, further studies will be needed to evaluate how the MB distributes at anatomical sites of interest, such as the lungs in the case of SARS-CoV-2 infection. The plug-and-play nature of the Multabody also lends itself to exploring alternate half-life extending moieties other than the Fc if bioavailability is the only desired trait absent of effector functions e.g., human serum albumin ${ }^{43}$, or binding moieties that bind human serum albumin $^{44,45}$.

Different increases in neutralization potency were observed for different $\mathrm{mAb}$ sequences tested on the MB against SARS-CoV-2. This suggests that the ability of the MB to enhance potency may depend on epitope location on the Spike, or the geometry of how the Fabs engage the antigen to achieve neutralization. The fact that the neutralization of two out of 20 SARS-CoV-2 RBD binders were not rescued by the $\mathrm{MB}$ platform suggests limitations based on mAb sequences and binding properties alone. Nevertheless, the capacity of the $\mathrm{MB}$ to transform avidity into neutralization potency across a range of epitope specificities on the SARS-CoV-2 Spike highlights the potential for using this technology broadly. It will be interesting to explore the potencyenhancement capacity of the MB platform against viruses with low surface spike density like HIV-1 ${ }^{46}$, or against other targets like the tumor necrosis factor receptor superfamily, where bivalency of conventional antibodies limits their efficient activation ${ }^{47}$.

Virus escape can arise in response to selective pressure from treatments or during natural selection. A conventional approach to combat escape mutants is the use of antibody cocktails targeting different epitopes. MBs showed a lower susceptibility to S mutations in comparison to their parental IgGs, presumably because the loss in affinity was compensated by enhanced binding avidity. Hence, when used in cocktails, the MB overcame viral sequence variability with exceptional potency. In addition, the split MB design allows combination of multiple antibody specificities within a single multimerized molecule resulting in similar potency and breadth as the $\mathrm{MB}$ cocktails. Importantly, the B.1.351 variant of concern that can escape the neutralization of several $\mathrm{mAbs}^{21-23}$ is neutralized with high potency by a trispecific Multabody, thus further highlighting the capacity of these molecules to resist viral escape. Multi-specificity within the same particle could offer additional advantages such as intra-S avidity and synergy for the right combination of mAbs, setting the stage for further investigation of different combinations of mAb specificities on the MB. Avidity and multi-specificity could also be leveraged to deliver a single molecule that neutralizes potently across viral genera, such as betacoronaviruses. 

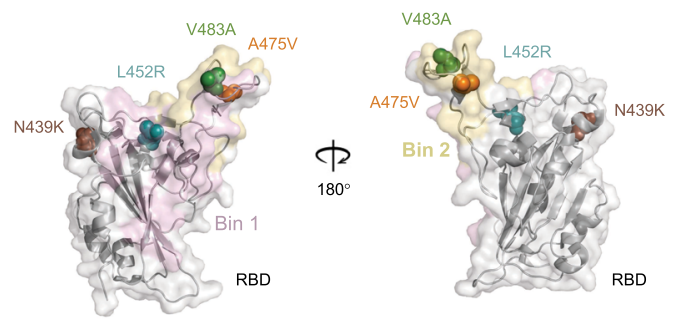

d

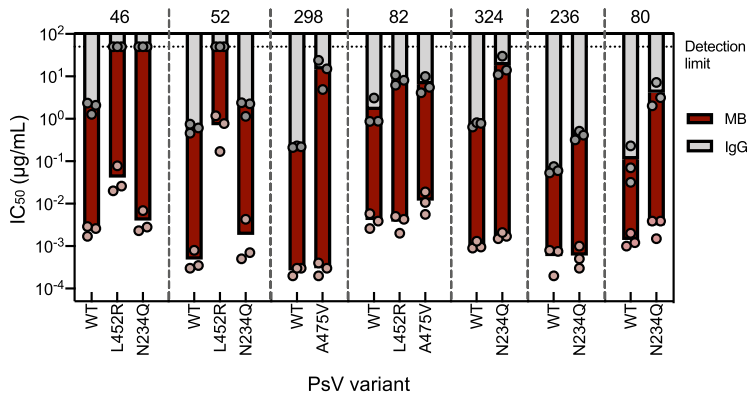

g

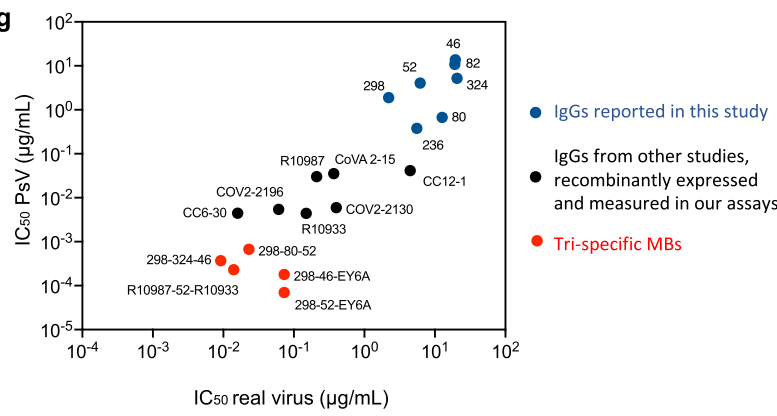

b

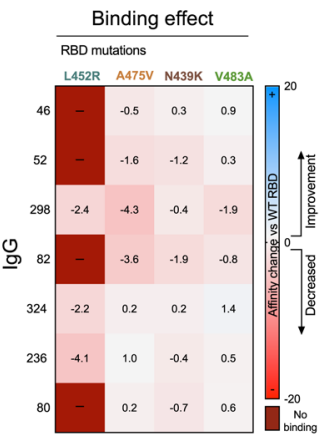

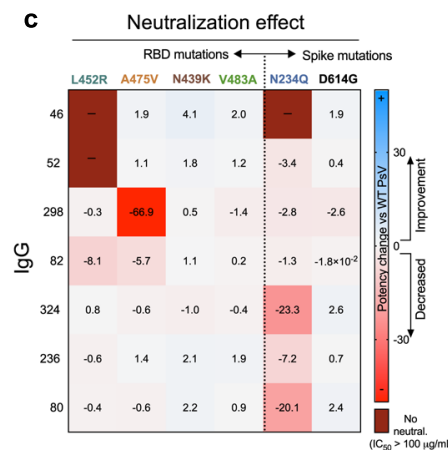

e Combination: $298-324-46$

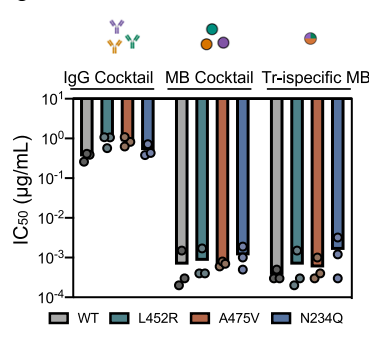

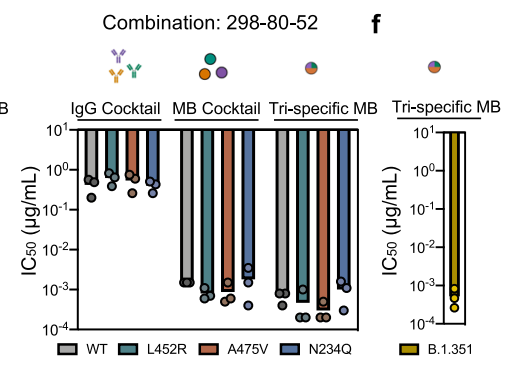

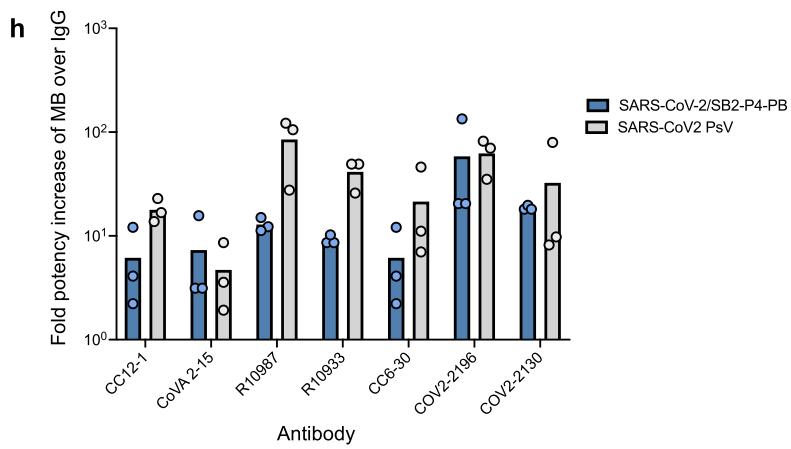

Fig. 6 Multabodies overcome SARS-CoV-2 sequence diversity. a Cartoon representation of the RBD showing four naturally occurring mutations as spheres. The epitopes of mAbs 52 (light pink) and 298 (yellow) are shown as representative epitopes of each bin. $\mathbf{b}$ Affinity and $\mathbf{c} I C_{50}$ fold-change comparison between WT and mutated RBD and PsV, respectively. $\mathbf{d}$ Neutralization potency of lgG (gray bars) vs MB (dark red bars) against SARS-CoV-2 PsV variants in comparison to WT PsV. e Neutralization potency comparison of two lgG cocktails (three lgGs), monospecific MB cocktails (three MBs) and tri-specific MBs against WT SARS-CoV-2 PsV and variants. mAbs sensitive to one or more PsV variants (d) were selected to generate the cocktails and the tri-specific MBs. $\mathbf{f}$ Neutralization potency of the tri-specific 298-80-52 MB against SARS-CoV-2 B.1.351 PsV variant. $\mathbf{g} I \mathrm{C}_{50}$ values in PsV ( $y$-axis) and replication competent SARS-CoV-2 virus (SB2-P4-PB: $x$-axis) demonstrating the ability of tri-specific MBs (red) to enhance potency across a wide range of $\mathrm{mAb}$ characteristics (blue and black). $\mathbf{h} \mathrm{IC}_{50}$ values fold increase upon multimerization. The mean of three biological replicates is shown in (b-h). Source data of panels $\mathbf{d}-\mathbf{h}$ are provided as a Source Data file.

Overall, the MB platform provides a tool to surpass antibody affinity limits and generate broad and potent neutralizing molecules while by-passing extensive antibody discovery or engineering efforts. This platform is an example of how binding avidity can be leveraged to accelerate the timeline to discovery of the most potent biologics against infectious diseases of global health importance.

\section{Methods}

Protein expression and purification. Genes encoding VHH-human apoferritin fusion, Fc fusions, Fabs, IgG, and RBD mutants were synthesized and cloned by GeneArt (Life Technologies) into the pcDNA3.4 expression vector. All constructs were expressed transiently in HEK $293 \mathrm{~F}$ cells (Thermo Fisher Scientific) at a density of $0.8 \times 10^{6}$ cells $/ \mathrm{mL}$ with $50 \mu \mathrm{g}$ of DNA per $200 \mathrm{~mL}$ of cells using FectoPRO (Polyplus Transfections) in a 1:1 ratio unless specified otherwise. After 6-7 days of incubation at $125 \mathrm{rpm}$ oscillation at $37^{\circ} \mathrm{C}, 8 \% \mathrm{CO}_{2}$, and $70 \%$ humidity in a Multitron Pro shaker (Infors HT), cell suspensions were harvested by centrifugation at $5000 \times g$ for $15 \mathrm{~min}$ and supernatants were filtered through a $0.22 \mu \mathrm{m}$ Steritop filter (EMD Millipore). Fabs and IgGs were transiently expressed by cotransfecting $90 \mu \mathrm{g}$ of the LC and the $\mathrm{HC}$ in a 1:2 ratio and purified using
KappaSelect affinity column (GE Healthcare) and HiTrap Protein A HP column (GE Healthcare), respectively with $100 \mathrm{mM}$ glycine $\mathrm{pH} 2.2$ as the elution buffer.

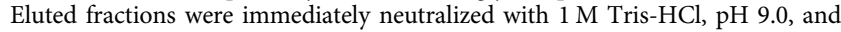
further purified using a Superdex 200 Increase size exclusion column (GE

Healthcare). Fc fusions of ACE2 and VHH-72 were purified the same way as IgGs. The VHH-72 apoferritin fusion was purified by hydrophobic interaction chromatography using a HiTrap Phenyl HP column and the eluted fraction was loaded onto a Superose 6 10/300 GL size exclusion column (GE Heathcare) in $20 \mathrm{mM}$ sodium phosphate $\mathrm{pH} 8.0,150 \mathrm{mM} \mathrm{NaCl}$. Wild type (BEI NR52309) and mutant RBDs, the prefusion S ectodomain (BEI NR52394) and $\mathrm{Fc}$ receptors ( $\mathrm{FcRn}$ and Fc $\gamma$ RI) from mouse and human were purified using a HisTrap Ni-NTA column (GE Healthcare). Ni-NTA purification was followed by Superose 6 in the case of the $\mathrm{S}$ trimer and Superdex 200 Increase size exclusion columns (GE Heathcare) in the case of the RBD and Fc receptors, in all cases in $20 \mathrm{mM}$ phosphate $\mathrm{pH} 8.0,150$ $\mathrm{mM} \mathrm{NaCl}$ buffer.

Design, expression, and purification of Multabodies. All molecules referred herein as Multabodies contain scFab and scFc fragments. The scFabs and scFc polypeptide constructs were generated using a 70 amino acid flexible linker $\left[(\mathrm{GGGGS})_{\mathrm{x} 14}\right]$ to generate heterodimers and homodimer fragments, respectively. Specifically, the C terminus of the Fab light chain is fused, through the linker, to the 
$\mathrm{N}$ terminus of the Fab heavy chain. In the case of the $\mathrm{scFc}$, the two single Fc chains that form the functional homodimer $\mathrm{Fc}$ were fused in tandem. The individual domains are fused to apoferritin monomers with a 25 amino acid linker: $(\text { GGGGS) })_{x 5}$. Genes encoding scFab and $\mathrm{scFc}$ fragments linked to half apoferritin were generated by deletion of residues 1 to 90 (C-Ferritin) and 91 to 175 (NFerritin) of the light chain of human apoferritin. Transient transfection of the Multabodies in HEK 293F cells were obtained by mixing $66 \mu \mathrm{g}$ of the plasmids scFab-human apoferritin: scFc-human N-Ferritin: scFab-C-Ferritin in a 2:1:1 ratio. Addition of scFab-human apoferritin allowed efficient Multabody assembly and increased the number of Fab's compared to Fc's in the final molecule, thus favoring Fab avidity over Fc avidity. In the case of multi-specific Multabodies, a 4:2:1:1 ratio of scFab1-human apoferritin: scFc-human N-Ferritin: scFab2-C-Ferritin: scFab3C-Ferritin was used. The DNA mixture was filtered and incubated at room temperature (RT) with $66 \mu \mathrm{l}$ of FectoPRO before adding to the cell culture. Split Multabodies were purified by affinity chromatography using a HiTrap Protein A HP column (GE Healthcare) with $20 \mathrm{mM}$ Tris $\mathrm{pH} 8.0,3 \mathrm{M} \mathrm{MgCl}_{2}$ and $10 \%$ glycerol elution buffer. Fractions containing the protein were concentrated and further purified by gel filtration on a Superose 6 10/300 GL column (GE Healthcare).

Negative-stain electron microscopy. Three microliters of Multabody at a concentration approximately of $0.02 \mathrm{mg} / \mathrm{mL}$ was placed on the surface of a carboncoated copper grid that had previously been glow-discharged in air for $15 \mathrm{~s}$, allowed to adsorb for $30 \mathrm{~s}$, and stained with $3 \mu \mathrm{L}$ of $2 \%$ uranyl formate. Excess stain was removed immediately from the grid using Whatman No. 1 filter paper and an additional $3 \mu \mathrm{L}$ of $2 \%$ uranyl formate was added for $20 \mathrm{~s}$. Grids were imaged with a FEI Tecnai T20 electron microscope operating at $200 \mathrm{kV}$ and equipped with an Orius charge-coupled device (CCD) camera (Gatan Inc).

Biolayer interferometry. Direct binding kinetics measurements were conducted using an Octet RED96 BLI system (Sartorius ForteBio) in PBS pH 7.4, 0.01\% BSA, and $0.002 \%$ Tween at $25^{\circ} \mathrm{C}$. His-tagged RBD, SARS-CoV-2 Spike was loaded onto Ni-NTA (NTA) biosensors (Sartorius ForteBio) to reach a BLI signal response of $0.8 \mathrm{~nm}$. Association rates were measured by transferring the loaded biosensors to wells containing a two-fold dilution series from 250 to $8 \mathrm{nM}$ (Fabs), 125 to $4 \mathrm{nM}$ (IgG), and 16 to $0.5 \mathrm{nM}(\mathrm{MB})$. Dissociation rates were measured by dipping the biosensors into buffer-containing wells. The duration of each step was $180 \mathrm{~s}$. Fc characterization in the split Multabody design was assessed by measuring binding to $\mathrm{hFc} \gamma \mathrm{RI}$ and $\mathrm{hFcRn}$ loaded onto Ni-NTA (NTA) biosensors following the experimental conditions and concentration ranges indicated above. To probe the theoretical capacity of the Multabodies to undergo endosomal recycling, binding to the hFcRn $\beta 2$-microglobulin complex was measured at physiological (7.4) and endosomal (5.6) $\mathrm{pH}$. Similarly, Fc characterization of the mouse surrogate MB was assessed by measuring binding to $\mathrm{mFc} \gamma \mathrm{RI}$ and $\mathrm{mFcRn}$, pre-immobilized onto NiNTA (NTA) biosensors. Two-fold dilution series from 100 to $3 \mathrm{nM}$ (IgG) and 10 to $0.3 \mathrm{nM}(\mathrm{MB})$ were used. Analysis of the sensograms was performed using the Octet software, with a 1:1 fit model. Competition assays were performed in a two-step binding process. Ni-NTA biosensors preloaded with His-tagged RBD were first dipped into wells containing the primary antibody at $50 \mu \mathrm{g} / \mathrm{mL}$ for $180 \mathrm{~s}$. After a 30 $s$ baseline period, the sensors were dipped into wells containing the second antibody at $50 \mu \mathrm{g} / \mathrm{ml}$ for an additional $300 \mathrm{~s}$. All incubation steps were performed in PBS pH 7.4, $0.01 \% \mathrm{BSA}$, and $0.002 \%$ Tween at $25^{\circ} \mathrm{C}$. ACE2-Fc was used to map $\mathrm{mAb}$ binding to the receptor binding site.

Dynamic light scattering. The Rh of the Multabody was determined by dynamic light scattering (DLS) using a DynaPro Plate Reader III (Wyatt Technology). About $20 \mu \mathrm{L}$ of the Multabody at a concentration of $1 \mathrm{mg} / \mathrm{mL}$ was added to a 384-well black, clear bottom plate (Corning) and measured at a fixed temperature of $25^{\circ} \mathrm{C}$ with a duration of $5 \mathrm{~s}$ per read. Particle size determination and polydispersity were obtained from the accumulation of five reads using the Dynamics software (Wyatt Technology).

Aggregation temperature. Aggregation temperature $\left(T_{\mathrm{agg}}\right)$ of the Multabodies and parental IgGs were determined using a UNit instrument (Unchained Labs). Samples were concentrated to $1.0 \mathrm{mg} / \mathrm{mL}$ and subjected to a thermal ramp from 25 to $95^{\circ} \mathrm{C}$ with $1{ }^{\circ} \mathrm{C}$ increments. $T_{\text {agg }}$ was determined as the temperature at which $50 \%$ increase in the static light scattering at a $266 \mathrm{~nm}$ wavelength relative to baseline was observed (i.e., the maximum value of the differential curve). The average and the standard error of two independent measurements were calculated using the UNit analysis software.

Pharmacokinetics and immunogenicity. A surrogate Multabody composed of the scFab and scFc fragments of mouse HD37 (anti-hCD19) IgG2a fused to the Nterminus of the light chain of mouse apoferritin (mFerritin) was used for the study. HD37 scFab-mFerritin: Fc-mFerritin: mFerritin in a 2:1:1 ratio was transfected and purified following the procedure described above. L234A, L235A, and P329G (LALAP) mutations were introduced in the mouse IgG2a Fc-construct to silence effector functions of the Multabody ${ }^{48}$. In vivo studies were performed using 12week-old male C57BL/6 mice purchased from Charles River (Strain code: 027), housed in individually-vented cages under $12 \mathrm{~h}$ light/dark cycle $(7 \mathrm{a} . \mathrm{m} . / 7 \mathrm{p} . \mathrm{m}$.) at a temperature of $21-23^{\circ} \mathrm{C}$ and a humidity of $40-55 \%$. All procedures were approved by the Local Animal Care Committee at the University of Toronto Scarborough. A single injection of $\sim 5 \mathrm{mg} / \mathrm{kg}$ of Multabodies or control samples (HD37 single chain IgG-IgG1 or IgG2a subtypes) and Helicobacter pylori ferritin (HpFerritin)-PfCSP malaria peptide in $200 \mu \mathrm{L}$ of PBS (pH 7.5) were subcutaneously injected. Blood samples were collected at multiple time points and serum samples were assessed for levels of circulating antibodies and anti-drug antibodies by ELISA. Briefly, 96-well Pierce Nickel Coated Plates (Thermo Fisher) were coated with $50 \mu \mathrm{L}$ at $0.5 \mu \mathrm{g} / \mathrm{ml}$ of the $\mathrm{His}_{6 \mathrm{x}}$-tagged antigen hCD19 to determine circulating HD37-specific concentrations using reagent-specific standard curves for IgGs and Multabodies. HRPProteinA (Invitrogen) was used to detect the levels of IgG/MBs bound (dilution 1:10,000). For anti-drug-antibody determination, Nunc MaxiSorp plates (Biolegend) were coated with a 12-mer HD37 scFab-mFerritin or with the HpFerritinPfCSP malaria peptide. 1:100 sera dilution was incubated for $1 \mathrm{~h}$ at RT and further develop using HRP-ProteinA (Invitrogen) as a secondary molecule (dilution $1: 10,000)$. The chemiluminescence signal at $450 \mathrm{~nm}$ was quantified using a Synergy Neo2 Multi-Mode Assay Microplate Reader (Biotek Instruments).

Biodistribution. Eight-week-old male BALB/c mice were purchased from The Jackson Laboratory and housed in individually-vented caging. Mice were housed $14 \mathrm{~h}$ of light $/ 10 \mathrm{~h}$ dark with phased in dawn to dusk intensity, maximum at noon at a temperature of $20-21{ }^{\circ} \mathrm{C}$ and a humidity of $40-60 \%$. All procedures were approved by the Local Animal Care Committee at the University of Toronto. Multabodies composed of the scFab and scFc fragments of mouse HD37 IgG2a fused to the $\mathrm{N}$-terminus of mouse apoferritin light chain was used for this study. HD37 IgG2a Multabody or control samples (HD37 single chain IgG2a) were fluorescently conjugated with Alexa-647 using Alexa Fluor ${ }^{\mathrm{TM}} 647$ Antibody Labeling kit (Invitrogen) as per the manufacturer's instruction. The $15 \mathrm{~nm}$ gold nanoparticles labeled with Alexa Fluor ${ }^{\mathrm{TM}} 647$ were purchased from Creative Diagnostics (GFLV-15). PerkinElmer IVIS Spectrum (PerkinElmer) was used to conduct noninvasive biodistribution experiments. $\mathrm{BALB} / \mathrm{c}$ mice were injected subcutaneously into the loose skin over the shoulders with $\sim 5 \mathrm{mg} / \mathrm{kg}$ of the MB, HD37 IgG2a, or gold nanoparticles in $200 \mu \mathrm{L}$ of PBS (pH 7.5) and imaged at time $0,1 \mathrm{~h}, 6 \mathrm{~h}, 24 \mathrm{~h}, 2,3,4,8$, and 11 days following injection. Prior to imaging, mice were placed in an anesthesia induction chamber containing a mixture of isoflurane and oxygen for $1 \mathrm{~min}$. Anesthetized mice were then placed in the prone position at the center of a built-in heated docking system within the IVIS imaging system (maintained at $37^{\circ} \mathrm{C}$ and supplied with a mixture of isoflurane and oxygen). For whole body $2 \mathrm{D}$ imaging, mice were imaged for $1-2 \mathrm{~s}$ (excitation $640 \mathrm{~nm}$ and emission $680 \mathrm{~nm}$ ) inside the imaging system. Data were analyzed using the IVIS software (Living Image Software for IVIS). After confirming the fluorescent signal from $2 \mathrm{D}$ epi-illumination images, 3D transilluminating fluorescence imaging tomography (FLIT) was performed on regions of interest using a built-in scan field of $3 \times 3$ or $3 \times 4$ transillumination positions. A series of $2 \mathrm{D}$ fluorescent surface radiance images were taken at various transillumination positions using an excitation of 640 and $680 \mathrm{~nm}$ emission. A series of CT scans were also taken at the corresponding positions. A 3D distribution map of the fluorescent signal was reconstructed by combining fluorescent signal and CT scans. Resulting 3D fluorescent images were thresholded based on the 3D images of PBS injected mice taken at the corresponding body positions. Images were mapped to the rainbow LUT in the IVIS software, with the upper end of the color scale set to $50 \mathrm{pmol} \mathrm{M}^{-1} \mathrm{~cm}^{-1}$ for mice injected with gold nanoparticles, and $1000 \mathrm{pmol} \mathrm{M}^{-1} \mathrm{~cm}^{-1}$ for $\mathrm{MB}$ and IgG2a injected mice, to allow for better visualization of biodistribution over the time course. A mouse organ registration feature of the IVIS software was used as a general guideline for assessing the sample body locations from $3 \mathrm{D}$ images.

Panning of phage libraries against the RBD of SARS-CoV-2. The commercial SuperHuman 2.0 Phage library (Distributed Bio/Charles River Laboratories) was used to identify monoclonal antibody binders to the SARS-CoV-2 RBD. For this purpose, an RBD-Fc-Avi tag construct of the SARS-CoV-2 was expressed in the EXPi-293 mammalian expression system. This protein was subsequently purified by protein G Dynabeads, biotinylated and quality-controlled for biotinylation and binding to ACE2 recombinant protein (Sino Biologics Inc). The SuperHuman 2.0 Phage library $\left(5 \times 10^{12}\right)$ was heated for $10 \mathrm{~min}$ at $72^{\circ} \mathrm{C}$ and de-selected against Protein G Dynabeads ${ }^{\mathrm{TM}}$ (Invitrogen), M-280 Streptavidin Dynabeads ${ }^{\mathrm{TM}}$ (Invitrogen), Histone from Calf Thymus (Sigma), Human IgG (Sigma) and ssDNABiotin NNK from Integrated DNA Technologies and DNA-Biotin NNK from Integrated DNA Technologies. Next, the library was panned against the RBDcaptured by M-280 Streptavidin Dynabeads ${ }^{\mathrm{TM}}$ using an automated protocol on Kingfisher FLEX (Thermofisher). Selected phages were acid eluted from the beads and neutralized using Tris-HCl pH 7.9 (Teknova). ER2738 cells were infected with the neutralized phage pools at $\mathrm{OD}_{600}=0.5$ at a $1: 10$ ratio and after $40 \mathrm{~min}$ incubation at $37^{\circ} \mathrm{C}$ and $100 \mathrm{rpm}$, the phage pools were centrifuged and incubated on agar with antibiotic selection overnight at $30^{\circ} \mathrm{C}$. The rescued phages were precipitated by PEG and subjected to three additional rounds of soluble-phase automated panning. PBST/1\% BSA buffer and/or PBS/1\% BSA was used in the deselection, washes and selection rounds. 


\section{Screening of anti-SARS-CoV-2 scFvs in bacterial PPE with SARS-CoV-2 RBD.} Anti-SARS-CoV-2 RBD scFvs selected from phage display were expressed and screened using high-throughput surface plasmon resonance (SPR) on Carterra LSA Array SPR instrument (Carterra) equipped with HC200M sensor chip (Carterra) at $25^{\circ} \mathrm{C}$. A V5 epitope tag was added to the scFv to enable capture via immobilized anti-V5 antibody (Abcam, Cambridge, MA) that was pre-immobilized on the chip surface by standard amine-coupling. Briefly: the chip surface was first activated by $10 \mathrm{~min}$ injection of a 1:1:1 (v/v/v) mixture of 0.4 M 1-ethyl-3-(3-dimethylaminopropyl) carbodiimide hydrochloride (EDC), $0.1 \mathrm{M} \mathrm{N}$-hydroxysulfosuccinimide (sNHS) and 0.1 M 2-( $N$-morpholino) ethanesulfonic acid (MES) pH 5.5. Then, 50 $\mu \mathrm{g} / \mathrm{ml}$ of anti-V5 tag antibody prepared in $10 \mathrm{mM}$ sodium acetate $\mathrm{pH} 4.3$ was coupled for $14 \mathrm{~min}$ and the excess reactive esters were blocked with $1 \mathrm{M}$ ethanolamine $\mathrm{HCl} \mathrm{pH} 8.5$ during a $10 \mathrm{~min}$ injection. For screening, a 384-ligand array comprising of crude bacterial periplasmic extracts (PPE) containing the scFvs (one spot per $\mathrm{scFv}$ ) was prepared. Each extract was prepared at a twofold dilution in running buffer (10 mM HEPES $\mathrm{pH} 7.4,150 \mathrm{mM} \mathrm{NaCl}, 3 \mathrm{mM} \mathrm{EDTA}$, and $0.01 \%$ (v/ v) Tween-20 (HBSTE)) and printed on the anti-V5 surface for $15 \mathrm{~min}$. SARS-CoV2 RBD Avi Tev His tagged was then prepared at 0, 3.7, 11.1, 33.3, 100, 37, and 300 $\mathrm{nM}$ in $10 \mathrm{mM}$ HEPES pH 7.4, $150 \mathrm{mM} \mathrm{NaCl}$, and $0.01 \%$ (v/v) Tween-20 (HBST) supplemented with $0.5 \mathrm{mg} / \mathrm{ml} \mathrm{BSA}$ and injected as analyte for $5 \mathrm{~min}$ with a $15 \mathrm{~min}$ dissociation time. Samples were injected in ascending concentration without any regeneration step. Binding data from the local reference spots was used to subtracted signal from the active spots and the nearest buffer blank analyte responses were subtracted to double-reference the data. The double-referenced data were fitted to a simple 1:1 Langmuir binding model in Carterra's Kinetic Inspection Tool (version Oct. 2019). Twenty medium-affinity binders from phage display screening were selected for the present study.

Pseudovirus production and neutralization. SARS-CoV-2 pseudotyped viruses (PsV) were generated using an HIV-based lentiviral system ${ }^{49}$ with few modifications. Briefly, 293T cells were co-transfected with a lentiviral backbone encoding the luciferase reporter gene (BEI NR52516), a plasmid expressing the Spike (BEI NR52310) and plasmids encoding the HIV structural and regulatory proteins Tat (BEI NR52518), Gag-pol (BEI NR52517), and Rev (BEI NR52519) using BioT transfection reagent (Bioland Scientific) and following the manufacturer's instructions. $24 \mathrm{~h}$ post transfection at $37^{\circ} \mathrm{C}, 5 \mathrm{mM}$ sodium butyrate was added to the media and the cells were incubated for an additional $24-30 \mathrm{~h}$ at $30^{\circ} \mathrm{C}$. SARSCoV-2 Spike mutant D614G was kindly provided by D.R. Burton (The Scripps Research Institute), SARS-COV-2 PsV variant B.1.351 was kindly provided by D.D Ho (Columbia University) and the rest of the PsV mutants were generated using the KOD-Plus mutagenesis kit (Toyobo, Osaka, Japan) using primers described in Table S4. PsV particles were harvested, passed through $0.45 \mu \mathrm{m}$ pore sterile filters and finally concentrated using a $100 \mathrm{~K}$ Amicon (Merck Millipore Amicon-Ultra 2.0 Centrifugal Filter Units).

Neutralization was determined in a single-cycle neutralization assay using 293T-ACE2 cells (BEI NR52511) and HeLa-ACE2 cells (kindly provided by D.R. Burton; The Scripps Research Institute). Cells were seeded the day before the experiment at a density of $10,000 \mathrm{cells} /$ well in a $100 \mu \mathrm{l}$ volume. In the case of $293 \mathrm{~T}$ cells, plates where pre-coated with poly-L-lysine (Sigma-Aldrich). The day of the experiment, $50 \mu \mathrm{l}$ of serially diluted IgGs and MB samples were incubated with $50 \mu \mathrm{l}$ of PsV for $1 \mathrm{~h}$ at $37^{\circ} \mathrm{C}$. After $1 \mathrm{~h}$ incubation, the incubated volume was added to the cells and incubated for $48 \mathrm{~h}$. PsV neutralization was monitored by adding 50 $\mu \mathrm{l}$ Britelite plus reagent (PerkinElmer) to $50 \mu \mathrm{l}$ of the cells and after $2 \mathrm{~min}$ incubation, the volume was transferred to a 96-well white plate (Sigma-Aldrich) and the luminescence in relative light units (RLUs) was measured using a Synergy Neo2 Multi-Mode Assay Microplate Reader (Biotek Instruments). Two to three biological replicates with two technical replicates each were performed. $\mathrm{IC}_{50}$ fold increase was calculated as:

$$
\operatorname{IgG~IC}{ }_{50}(\mu \mathrm{g} / \mathrm{mL}) / \mathrm{MB} \mathrm{IC}_{50}(\mu \mathrm{g} / \mathrm{mL})
$$

Authentic virus neutralization. VeroE6 cells were seeded in a $96 \mathrm{~F}$ plate at a concentration of 30,000/well in DMEM supplemented with $100 \mathrm{U}$ Penicillin, $100 \mathrm{U}$ Streptomycin, and $10 \%$ FBS. Cells were allowed to adhere to the plate and rest overnight. After $24 \mathrm{~h}$, fivefold serial dilutions of the IgG and MB samples were prepared in DMEM supplemented with $100 \mathrm{U}$ Penicillin and $100 \mathrm{U}$ Streptomycin in a $96 \mathrm{R}$ plate in quadruplicates $(25 \mu \mathrm{L} /$ well). About $25 \mu \mathrm{L}$ of SARS-CoV-2/SB2$\mathrm{P} 4-\mathrm{PB}^{50}$ Clone 1 was added to each well at $100 \mathrm{TCID} /$ well and incubated for $1 \mathrm{~h}$ at $37^{\circ} \mathrm{C}$ with shaking every $15 \mathrm{~min}$. After co-culturing, the media from the VeroE6 plate was removed, and $50 \mu \mathrm{L}$ antibody-virus sample was used to inoculate VeroE6 cells in quadruplicates for $1 \mathrm{~h}$ at $37^{\circ} \mathrm{C}, 5 \% \mathrm{CO}_{2}$, shaking every $15 \mathrm{~min}$. After $1 \mathrm{~h}$ inoculation, the inoculum was removed and $200 \mu \mathrm{L}$ of fresh DMEM supplemented with 100U Penicillin, 100U Streptomycin, and 2\% FBS was added to each well. The plates were further incubated for 5 days. The cytopathic effect (CPE) was monitored and PRISM was used to calculate IC $_{50}$ values. Three biological replicates with four technical replicates each were performed.

Cross-linking of Spike protein with Fabs 80, 298, and 324. About $100 \mu \mathrm{g}$ of Spike trimer was mixed with 2x molar excess of Fab 80, 298, or 324 in $20 \mathrm{mM}$
HEPES pH 7.0 and $150 \mathrm{mM} \mathrm{NaCl}$. Proteins were crosslinked by addition of $0.075 \%$ (v/v) glutaraldehyde (Sigma Aldrich) and incubated at RT for $120 \mathrm{~min}$. Complexe were purified via size exclusion chromatography (Superose6 Increase 10/300 GL, GE Healthcare), concentrated to $0.5 \mathrm{mg} / \mathrm{mL}$ and directly used for cryo-EM grid preparation.

Cross-linking of Fab 46-RBD complex. About $100 \mu \mathrm{g}$ of Fab 46 was mixed with $2 \mathrm{x}$ molar excess of RBD in $20 \mathrm{mM}$ HEPES pH 7.0 and $150 \mathrm{mM} \mathrm{NaCl}$. The complex was crosslinked by addition of $0.05 \%(\mathrm{v} / \mathrm{v})$ glutaraldehyde (Sigma Aldrich) and incubated at RT for $45 \mathrm{~min}$. The cross-linked complex was purified via size exclusion chromatography (Superdex 200 Increase 10/300 GL, GE Healthcare), concentrated to $2.0 \mathrm{mg} / \mathrm{ml}$ and directly used for cryo-EM grid preparation.

Cryo-EM data collection and image processing. Three microliters of sample was deposited on holey gold grids prepared in-house ${ }^{51}$, which were glow-discharged in air for $15 \mathrm{~s}$ with a PELCO easiGlow (Ted Pella) before use. Sample was blotted for $6 \mathrm{~s}$ with a modified FEI Mark III Vitrobot (maintained at $4{ }^{\circ} \mathrm{C}$ and $100 \%$ humidity) using an offset of -5 , and subsequently plunge-frozen in a mixture of liquid ethane and propane. Data were acquired at $300 \mathrm{kV}$ with a Thermo Fisher Scientific Titan Krios G3 electron microscope and prototype Falcon 4 camera operating in electron counting mode at 250 frames/s. Movies were collected for $9.6 \mathrm{~s}$ with 29 exposure fractions, a camera exposure rate of $\sim 5 \mathrm{e}^{-} / \mathrm{pix} / \mathrm{s}$, and total specimen exposure of $\sim 44 \mathrm{e}^{-} / \AA^{2}$. No objective aperture was used. The pixel size was calibrated at $1.03 \AA /$ pixel from a gold diffraction standard. The microscope was automated with the EPU software package and data collection were monitored with cryoSPARC Live ${ }^{52}$

To overcome preferred orientation encountered with some of the samples, tilted data collection was employed ${ }^{53}$. For the Spike-Fab 80 complex, $8200^{\circ}$ tilted movies and $279040^{\circ}$ tilted movies were collected. For the Spike-Fab 298 complex, $42590^{\circ}$ tilted movies and $351340^{\circ}$ tilted movies were collected. For the Spike-Fab 324 complex, $10980^{\circ}$ tilted movies and $338040^{\circ}$ tilted movies were collected. For the RBD-Fab 46 complex, $47220^{\circ}$ tilted movies were collected. For $0^{\circ}$ tilted movies, cryoSPARC patch motion correction was performed. For $40^{\circ}$ tilted movies, Relion MotionCorr ${ }^{54,55}$ was used. Micrographs were then imported into cryoSPARC and patch CTF estimation was performed. Templates generated from 2D classification during the cryoSPARC Live session were used for template selection of particles. 2D classification was used to remove junk particle images, resulting in a dataset of 80,951 particle images for the Spike-Fab 80 complex, 203,138 particle images for the Spike-Fab 298 complex, 64,365 particle images for the Spike-Fab 324 complex, and 2,143,629 particle images for the RBD-Fab 46 complex. Multiple rounds of multi-class ab initio refinement were used to clean up the particle image stacks, and homogeneous refinement was used to obtain consensus structures. For tilted particles, particle polishing was done within Relion at this stage and reimported back into cryoSPARC. For the Spike-Fab complexes, extensive flexibility was observed. $3 \mathrm{D}$ variability analysis was performed ${ }^{56}$ and together with heterogeneous refinement used to classify out the different states present. Nonuniform refinement was then performed on the final set of particle images ${ }^{57}$. For the RBD-Fab 46 complex, cryoSPARC ab initio refinement with three classes was used iteratively to clean up the particle image stack. Thereafter, the particle image stack with refined Euler angles was brought into cisTEM for reconstruction ${ }^{58}$ to produce a $4.0 \AA$ resolution map. Transfer of data between Relion and cryoSPARC was done with pyem $^{59}$.

Crystallization and structure determination. A ternary complex of 52 Fab-298 Fab-RBD was obtained by mixing $200 \mu \mathrm{g}$ of RBD with $2 \mathrm{x}$ molar excess of each Fab in $20 \mathrm{mM}$ Tris $\mathrm{pH} 8.0,150 \mathrm{mM} \mathrm{NaCl}$, and subsequently purified via size exclusion chromatography (Superdex 200 Increase 10/300 GL, GE Healthcare). Fractions containing the complex were concentrated to $7.3 \mathrm{mg} / \mathrm{ml}$ and mixed in a $1: 1$ ratio with 20\% (w/v) 2-propanol, 20\% (w/v) PEG 4000, and $0.1 \mathrm{M}$ sodium citrate pH 5.6 Crystals appeared after $\sim 1$ day and were cryoprotected in $10 \%(\mathrm{v} / \mathrm{v})$ ethylene glycol before being flash-frozen in liquid nitrogen.

Data were collected on the 23-ID-D beamline at the Argonne National Laboratory Advanced Photon Source. The dataset was processed using $\mathrm{XDS}^{60}$ and XPREP. Phases were determined by molecular replacement using Phaser ${ }^{61}$ with CNTO88 Fab as a model for 52 Fab (PDB ID: 4DN3), 20358 Fab as a model for 298 Fab (PDB ID: 5CZX), and PDB ID: 6XDG as a search model for the RBD. Refinement of the structure was performed using phenix.refine ${ }^{62}$ and iterations of manual building in $\operatorname{Coot}^{63}$. PyMOL was utilized for structure analysis and figure rendering ${ }^{64}$. Access to all software was supported through SBGrid ${ }^{65}$. Representative electron density for the two Fab-RBD interfaces is shown in Fig. S7e, f.

Reporting summary. Further information on research design is available in the Nature Research Reporting Summary linked to this article.

\section{Data availability}

The electron microscopy maps have been deposited in the Electron Microscopy Data Bank (EMDB) with accession codes EMD-22738, EMD-22739, EMD-22740, and EMD 22741 (Table S1- https://doi.org/10.1101/2020.10.15.341636). The crystal structure of 
the 298-52-RBD complex (Table S2- https://doi.org/10.2210/pdb7K9Z/pdb) is available from the Protein Data Bank under accession PDB ID: 7K9Z. The sequences of the monoclonal antibodies used are provided with this paper (Supplementary Data 1). Additional PDB/EMDB entries were used throughout the manuscript to perform a comparative analysis of the different epitope bins targeted by mAbs. The entries used in this analysis are: REGN10933 (PDB ID: 6XDG), CV30 (PDB ID: 6XE1), C105 (PDB ID: 6XCM), COVA2-04 (PDB ID: 7JMO), COVA2-39 (PDB ID: 7JMP), CC12.1 (PDB ID: 6XC2), BD23 (PDB ID: 7BYR), B38 (PDB ID: 7BZ5), P2C-1F11 (PDB ID: 7BWJ), 2-4 (PDB ID: 6XEY), CB6 (PDB ID: 7C01), REGN10987 (PDB ID: 6XDG), S309 (PDB ID: 6WPS, 6WPT), EY6A (PDB ID: 6ZCZ), CR3022 (PDB ID: 6YLA), H014 (PDB ID: 7CAH), 4-8 (EMDB ID: 22159), 4A8 (PDB ID: 7C2L), and 2-43 (EMDB ID: 22275). All data supporting the findings of this study are available within the paper and its supplementary information files. Requests for material can be directed to Jean-Philippe Julien (jean-philippe.julien@sickkids.ca). All materials and reagents will be available upon establishing a material transfer agreement (MTA). Source data are provided with this paper.

Received: 13 January 2021; Accepted: 19 May 2021; Published online: 16 June 2021

\section{References}

1. Connor, E. M. Palivizumab, a humanized respiratory syncytial virus monoclonal antibody, reduces hospitalization from respiratory syncytial virus infection in high-risk infants. Pediatrics 102, 531-537 (1998).

2. Mulangu, S. et al. A randomized, controlled trial of Ebola virus disease therapeutics. N. Engl. J. Med. 381, 2293-2303 (2019).

3. Ju, B. et al. Human neutralizing antibodies elicited by SARS-CoV-2 infection. Nature 584, 115-119 (2020).

4. Liu, L. et al. Potent neutralizing antibodies against multiple epitopes on SARSCoV-2 spike. Nature 584, 450-456 (2020).

5. Wang, C. et al. A human monoclonal antibody blocking SARS-CoV-2 infection. Nat. Commun. 11, 2251 (2020).

6. Zost, S. J. et al. Potently neutralizing and protective human antibodies against SARS-CoV-2. Nature 584, 443-449 (2020).

7. Baum, A. et al. Antibody cocktail to SARS-CoV-2 spike protein prevents rapid mutational escape seen with individual antibodies. Science 369, 1014-1018 (2020).

8. Hansen, J. et al. Studies in humanized mice and convalescent humans yield a SARS-CoV-2 antibody cocktail. Science 369, 1010-1014 (2020).

9. Lv, Z. et al. Structural basis for neutralization of SARS-CoV-2 and SARS-CoV by a potent therapeutic antibody. Science 369, 1505-1509 (2020).

10. Tortorici, M. A. et al. Ultrapotent human antibodies protect against SARSCoV-2 challenge via multiple mechanisms. Science 370, 950-957 (2020).

11. Zhou, D. et al. Structural basis for the neutralization of SARS-CoV-2 by an antibody from a convalescent patient. Nat. Struct. Mol. Biol. 27, 950-958 (2020).

12. Cao, Y. et al. Potent neutralizing antibodies against SARS-CoV-2 identified by high-throughput single-cell sequencing of convalescent patients' B cells. Cell 182, 73-84 (2020).

13. Chi, X. et al. A neutralizing human antibody binds to the N-terminal domain of the Spike protein of SARS-CoV-2. Science 369, 650-655 (2020).

14. Seydoux, E. et al. Analysis of a SARS-CoV-2-infected individual reveals development of potent neutralizing antibodies with limited somatic mutation. Immunity 53, 98-105 (2020).

15. Pinto, D. et al. Cross-neutralization of SARS-CoV-2 by a human monoclonal SARS-CoV antibody. Nature 583, 290-295 (2020).

16. Barnes, C. O. et al. Structures of human antibodies bound to SARS-CoV-2 spike reveal common epitopes and recurrent features of antibodies. Cell 182, 828-842.e16 (2020).

17. Shi, R. et al. A human neutralizing antibody targets the receptor-binding site of SARS-CoV-2. Nature 584, 120-124 (2020).

18. Rogers, T. F. et al. Isolation of potent SARS-CoV-2 neutralizing antibodies and protection from disease in a small animal model. Science 369, 956-963 (2020).

19. Brouwer, P. J. M. et al. Potent neutralizing antibodies from COVID-19 patients define multiple targets of vulnerability. Science 369, 643-650 (2020).

20. Hoffmann, M. et al. SARS-CoV-2 cell entry depends on ACE2 and TMPRSS2 and is blocked by a clinically proven protease inhibitor. Cell 181, 271-280 (2020).

21. Wang, P. et al. Increased resistance of SARS-CoV-2 variant P.1 to antibody neutralization. Cell Host Microbe. 29, 747-751.e4 (2021).

22. $\mathrm{Wu}, \mathrm{K}$. et al. mRNA-1273 vaccine induces neutralizing antibodies against spike mutants from global SARS-CoV-2 variants. Preprint at bioRxiv (2021).

23. Wibmer, C. K. et al. SARS-CoV-2 501Y.V2 escapes neutralization by South African COVID-19 donor plasma. Nat. Med. 27, 622-625 (2021).
24. Rambaut, A. et al. Preliminary genomic characterisation of an emergent SARS-CoV-2 lineage in the UK defined by a novel set of spike mutations. Preprint at https://virological.org/t/preliminarygenomic-characterisation-ofan-emergent-sars-cov-2-lineage-in-the-uk-defined-by-a-novel-set-ofspikemutations/563 (2020).

25. Tegally, H. et al. Emergence and rapid spread of a new severe acute respiratory syndrome-related coronavirus 2 (SARS-CoV-2) lineage with multiple spike mutations in South Africa. Preprint at medRxiv https://doi.org/10.1101/ 2020.12.21.20248640 (2020).

26. Faria, N. R. et al. Genomics and epidemiology of the P.1 SARS-CoV-2 lineage in Manaus, Brazil. Science. 372, 815-821 (2021).

27. Naveca, F. et al. Phylogenetic relationship of SARS-CoV-2 sequences from Amazonas with emerging Brazilian variants harboring mutations E484K and N501Y in the spike protein. Preprint at https://virological.org/t/phylogeneticrelationship-of-sars-cov-2-sequences-from-amazonas-with-emergingbrazilian-variants-harboring-mutations-e484k-and-n501y-in-the-spikeprotein/585 (2021).

28. $\mathrm{Wu}, \mathrm{H}$. et al. Ultra-potent antibodies against respiratory syncytial virus: effects of binding kinetics and binding valence on viral neutralization. J. Mol. Biol. 350, 126-144 (2005).

29. Icenogle, J. et al. Neutralization of poliovirus by a monoclonal antibody: kinetics and stoichiometry. Virology 127, 412-425 (1983).

30. Cavacini, L. A., Emes, C. L., Power, J., Duval, M. \& Posner, M. R. Effect of antibody valency on interaction with cell-surface expressed HIV- 1 and viral neutralization. J. Immunol. 152, 2538-2545 (1994).

31. Wrapp, D. et al. Structural basis for potent neutralization of betacoronaviruses by single-domain camelid antibodies. Cell 181, 1004-1015.e15 (2020).

32. $\mathrm{Li}, \mathrm{W}$. et al. High potency of a bivalent human VH domain in SARS-CoV-2 animal models. Cell 183, 1-13 (2020).

33. Lawson, D. M. et al. Solving the structure of human $\mathrm{H}$ ferritin by genetically engineering intermolecular crystal contacts. Nature 349, 541-544 (1991).

34. Radomsky, M. L., Whaley, K. J., Cone, R. A. \& Saltzman, W. M. Macromolecules released from polymers: diffusion into unstirred fluids. Biomaterials 11, 619-624 (1990).

35. $\mathrm{Li}, \mathrm{Q}$. et al. The impact of mutations in SARS-CoV-2 spike on viral infectivity and antigenicity. Cell 182, 1284-1294 (2020).

36. Thomson, E. C. et al. Circulating SARS-CoV-2 spike N439K variants maintain fitness while evading antibody-mediated immunity. Cell. 185, 1171-1187.e20 (2021).

37. Korber, B. et al. Tracking changes in SARS-CoV-2 spike: evidence that D614G increases infectivity of the COVID-19 virus. Cell 182, 812-827 (2020).

38. Miller, K. et al. Design, construction, and in vitro analyses of multivalent antibodies. J. Immunol. 170, 4854-4861 (2003).

39. Kipriyanov, S. M. et al. Bispecific tandem diabody for tumor therapy with improved antigen binding and pharmacokinetics. J. Mol. Biol. 293, 41-56 (1999).

40. Kipriyanov, S. M. et al. Affinity enhancement of a recombinant antibody: formation of complexes with multiple valency by a single-chain Fv fragmentcore streptavidin fusion. Protein Eng. 9, 203-211 (1996).

41. Zhang, J. et al. Pentamerization of single-domain antibodies from phage libraries: a novel strategy for the rapid generation of high-avidity antibody reagents. J. Mol. Biol. 335, 49-56 (2004).

42. Hoffmann, M. A. G. et al. Nanoparticles presenting clusters of CD4 expose a universal vulnerability of HIV-1 by mimicking target cells. Proc. Natl Acad. Sci. USA 117, 18719-18728 (2020).

43. Sleep, D., Cameron, J. \& Evans, L. R. Albumin as a versatile platform for drug half-life extension. Biochim. Biophys. Acta 1830, 5526-5534 (2013)

44. van Faassen, H. et al. Serum albumin-binding VHHs with variable $\mathrm{pH}$ sensitivities enable tailored half-life extension of biologics. FASEB J. 34, 8155-8171 (2020).

45. Adams, R. et al. Extending the half-life of a fab fragment through generation of a humanized anti-human serum albumin Fv domain: an investigation into the correlation between affinity and serum half-life. MAbs 8, 1336-1346 (2016).

46. Zhu, P. et al. Electron tomography analysis of envelope glycoprotein trimers on HIV and simian immunodeficiency virus virions. Proc. Natl Acad. Sci. USA 100, 15812-15817 (2003)

47. Wajant, H. Principles of antibody-mediated TNF receptor activation. Cell Death Differ. 22, 1727-1741 (2015).

48. Schlothauer, T. et al. Novel human IgG1 and IgG4 Fc-engineered antibodies with completely abolished immune effector functions. Protein Eng. Des. Sel. 29, 457-466 (2016).

49. Crawford, K. H. D. et al. Protocol and reagents for pseudotyping lentiviral particles with SARS-CoV-2 spike protein for neutralization assays. Viruses 12, 513 (2020).

50. Banerjee, A. et al. Isolation, sequence, infectivity, and replication kinetics of severe acute respiratory syndrome coronavirus 2 . Emerg. Infect. Dis. 26, 2054-2063 (2020) 
51. Marr, C. R., Benlekbir, S. \& Rubinstein, J. L. Fabrication of carbon films with $\sim 500 \mathrm{~nm}$ holes for cryo-EM with a direct detector device. J. Struct. Biol. 185, 42-47 (2014)

52. Punjani, A., Rubinstein, J. L., Fleet, D. J. \& Brubaker, M. A. CryoSPARC: algorithms for rapid unsupervised cryo-EM structure determination. Nat. Methods 14, 290-296 (2017).

53. $\mathrm{Zi}$ Tan, Y. et al. Addressing preferred specimen orientation in single-particle cryo-EMthrough tilting. Nat. Methods 14, 793-796 (2017).

54. Zivanov, J. et al. New tools for automated high-resolution cryo-EM structure determination in RELION-3. Elife 9, e42166 (2018).

55. Scheres, S. H. W. RELION: implementation of a Bayesian approach to cryoEM structure determination. J. Struct. Biol. 180, 519-530 (2012).

56. Punjani, A. \& Fleet, D. J. 3D variability analysis: Resolving continuous flexibility and discrete heterogeneity from single particle cryo-EM. J. Struct. Biol. 213, 107702 (2021).

57. Punjani, A., Zhang, H. \& Fleet, D. J. Non-uniform refinement: adaptive regularization improves single-particle cryo-EM reconstruction. Nat. Methods. 17, 1214-1221 (2020).

58. Grant, T., Rohou, A. \& Grigorieff, N. CisTEM, user-friendly software for single-particle image processing. Elife 7, e35383 (2018).

59. Asarnow, D., Palovcak, E. \& Cheng, Y. asarnow/pyem: UCSF pyem v0. 5 (Zenodo, 2019).

60. Kabsch, W. et al. XDS. Acta Crystallogr. D. Biol. Crystallogr. 66, 125-132 (2010).

61. McCoy, A. J. et al. Phaser crystallographic software. J. Appl. Crystallogr. 40 658-674 (2007).

62. Adams, P. D. et al. PHENIX: a comprehensive Python-based system for macromolecular structure solution. Acta Crystallogr. D Biol. Crystallogr. 66 , 213-221 (2010).

63. Emsley, P., Lohkamp, B., Scott, W. G. \& Cowtan, K. Features and development of Coot. Acta Crystallogr. Sect. D Biol. Crystallogr. 66, 486-501 (2010).

64. The PyMol Molecular Graphics System, Versión 1.8 (Schrödinger, LLC., 2015).

65. Morin, A. et al. Collaboration gets the most out of software. Elife 2, e01456 (2013).

66. Lan, J. et al. Structure of the SARS-CoV-2 spike receptor-binding domain bound to the ACE2 receptor. Nature 581, 215-220 (2020).

67. Yuan, M. et al. A highly conserved cryptic epitope in the receptor binding domains of SARS-CoV-2 and SARS-CoV. Science 368, 630-633 (2020).

68. Piccoli, L. et al. Mapping neutralizing and immunodominant sites on the SARS-CoV-2 spike receptor-binding domain by structure-guided highresolution serology. Cell 183, 1024-1042.e21 (2020).

69. Hurlburt, N. K. et al. Structural basis for potent neutralization of SARS-CoV-2 and role of antibody affinity maturation. Nat. Commun. 11, 5413 (2020).

70. Barnes, C. O. et al. SARS-CoV-2 neutralizing antibody structures inform therapeutic strategies. Nature 588, 682-687 (2020).

71. Yuan, M. et al. Structural basis of a shared antibody response to SARS-CoV-2. Science 369, 1119-1123 (2020).

72. Hansen, J. et al. Studies in humanized mice and convalescent humans yield a SARS-CoV-2 antibody cocktail. Science 369, 1010-1014 (2020).

\section{Acknowledgements}

We thank F. Krammer for providing the WT SARS-CoV-2 Spike plasmid. We thank J.D. Bloom and A.C. Gingras for access to 293T-ACE2 cells and reagents to make the SARSCoV-2 PsV. We thank D.R. Burton for providing HeLa-ACE2 cells and the SARS-CoV-2 Spike mutant D614G. We are thankful to D.D. Ho for providing the SARS-COV-2 PsV variant B.1.351. We thank C. Pettus, J. Wang, D. Pineda, and K. Patel for their work panning the SuperHuman 2.0 library against the SARS-CoV-2 RBD and characterizing binders. We are grateful to A. Banerjee and K. Mossman for their contributions to the isolation of SARS-CoV-2 (SARS-CoV-2/SB2-P4-PB Clone 1). We thank J.M. Jorgensen and S. Popa for assistance with the Octet RED96 and Unit Instruments. The following reagents were produced under HHSN272201400008C and obtained through BEI Resources, NIAID, NIH: Vector pCAGGS containing the SARS-related Coronavirus 2, Wuhan-Hu-1 Spike glycoprotein RBD, NR-52309, Vector pCAGGS containing the SARS-related Coronavirus 2, Wuhan-Hu-1 Spike glycoprotein gene (soluble and stabilized), NR-52394. This work was supported by Natural Sciences and Engineering Research Council of Canada discovery grant 6280100058 (J.-P.J.), by operating grant PJ4-
169662 from the Canadian Institutes of Health Research (CIHR; B.T. and J.-P.J.), by COVID-19 Research Fund C-094-2424972-JULIEN (J.-P.J.) from the Province of Ontario Ministry of Colleges and Universities, by Bill and Melinda Gates Foundation INV-023398 (J.-P.J.) and by the Hospital for Sick Children Foundation. This research was also supported by the European Union's Horizon 2020 research and innovation program under Marie Sklodowska-Curie grant 790012 (E.R.), by a Hospital for Sick Children Restracomp Postdoctoral Fellowship (I.K. and C.B.A.), by a CIHR Postdoctora Fellowship (Y.Z.T.), by a NSERC postgraduate doctoral scholarship (T.Z.), by a Vanier Canada Graduate Scholarship (T.S.), by a CIHR Canada Graduate Scholarship- Master's Award (A.N.), by the CIFAR Azrieli Global Scholar program (J.-P.J.), by the Ontario Early Researcher Awards program (J.-P.J.), and by the Canada Research Chairs program (J.L.R., B.T., and J.-P.J.). Cryo-EM data were collected at the Toronto High Resolution High Throughput cryo-EM facility, biophysical data at the Structural \& Biophysical Core facility, and biodistribution data at the CFI 3D Facility at University of Toronto, all supported by the Canada Foundation for Innovation and Ontario Research Fund. X-ray diffraction experiments were performed at GM/CA@APS, which has been funded in whole or in part with federal funds from the National Cancer Institute (ACB-12002) and the National Institute of General Medical Sciences (AGM-12006). The Eiger $16 \mathrm{M}$ detector at GM/CA-XSD was funded by NIH grant S10 OD012289. This research used resources of the Advanced Photon Source, a US Department of Energy (DOE) Office of Science user facility operated for the DOE Office of Science by Argonne National Laboratory under contract DE-AC02-06CH11357.

\section{Author contributions}

E.R., B.T., and J.-P.J. conceived the research and designed the experiments; E.R., I.K., Y.Z T., S.B., H.C., T.Z., G.A.W., P.B., F.G., J.C.N., T.S., A.S., K.M., A.N., C.B.A., K.P., S.A.B., S.Y., and S.L.-C. performed experimental work. J.G., N.C.-H., S.M., and S.D.G.-O. provided critical reagents and expertize. E.R., I.K., Y.C.T., B.T., J.L.R., and J.-P.J. analyzed the data. E.R. and J.-P.J. wrote the manuscript with input from all authors.

\section{Competing interests}

The Hospital for Sick Children has applied for patents concerning SARS-CoV-2 antibodies and the Multabody platform technology that are related to this work. B.T. and J.-P.J. are founders of Radiant Biotherapeutics and are members of its Scientific Advisory Board. S.Y., S.L.C., and J.G. are employees of DistributedBio and may hold shares in DistributedBio. The remaining authors declare no competing interests.

\section{Additional information}

Supplementary information The online version contains supplementary material available at https://doi.org/10.1038/s41467-021-23825-2.

Correspondence and requests for materials should be addressed to J.-P.J.

Peer review information Nature Communications thanks the anonymous reviewers for their contribution to the peer review of this work. Peer reviewer reports are available.

Reprints and permission information is available at http://www.nature.com/reprints

Publisher's note Springer Nature remains neutral with regard to jurisdictional claims in published maps and institutional affiliations.

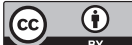

Open Access This article is licensed under a Creative Commons Attribution 4.0 International License, which permits use, sharing, adaptation, distribution and reproduction in any medium or format, as long as you give appropriate credit to the original author(s) and the source, provide a link to the Creative Commons license, and indicate if changes were made. The images or other third party material in this article are included in the article's Creative Commons license, unless indicated otherwise in a credit line to the material. If material is not included in the article's Creative Commons license and your intended use is not permitted by statutory regulation or exceeds the permitted use, you will need to obtain permission directly from the copyright holder. To view a copy of this license, visit http://creativecommons.org/ licenses/by/4.0/.

(C) The Author(s) 2021 